\title{
A NEW CHARACTERIZATION OF REGULARIZED BMO SPACES ON NON-HOMOGENEOUS SPACES AND ITS APPLICATIONS
}

\author{
Guoen Hu, Yan Meng* and Dachun Yang \\ Zhengzhou Information Science and Technology Institute, Department of Applied Mathematics \\ P.O. Box 1001-747, Zhengzhou 450002, P. R. China; guoenxx@yahoo.com.cn \\ Renmin University of China, School of Information \\ Beijing 100872, P. R. China; mengyan@ruc.edu.cn \\ Beijing Normal University, School of Mathematical Sciences \\ Laboratory of Mathematics and Complex Systems, Ministry of Education \\ Beijing 100875, P. R. China; dcyang@bnu.edu.cn
}

\begin{abstract}
Let $(\mathcal{X}, d, \mu)$ be a metric measure space and satisfy the so-called upper doubling condition and the geometrically doubling condition. Under this assumption, in this paper, the authors establish a new characterization of the space $\operatorname{RBMO}(\mu)$. As applications, the authors prove that the $L^{p}(\mu)$-boundedness with $p \in(1, \infty)$ of the Calderón-Zygmund operator is equivalent to its various endpoint estimates.
\end{abstract}

\section{Introduction}

The theory of singular integrals on classical Euclidean spaces has been proved to be a very fruitful part of analysis. Later, the results of the Calderón-Zygmund theory for singular integrals on classical Euclidean spaces have been extended to more general spaces under the assumption that the measure on the underlying space satisfies the doubling property (see (1.1) below). One of the most general settings for the Calderón-Zygmund theory is that of the space of homogeneous type in the sense of Coifman and Weiss [2]. A metric space $(\mathcal{X}, d)$ equipped with a nonnegative Borel measure $\mu$ is called a space of homogeneous type if $(\mathcal{X}, d, \mu)$ satisfies the following doubling condition: there exists a positive constant $C_{\mu}$ such that for any ball $B(x, r):=\{y \in \mathcal{X}: d(x, y)<r\}$ with $x \in \mathcal{X}$ and $r \in(0, \infty)$,

$$
\mu(B(x, 2 r)) \leq C_{\mu} \mu(B(x, r)) .
$$

In recent years, there has been significant progress in the study of the CalderónZygmund theory associated with non-doubling measures. To be precise, let $\mu$ be a non-negative Radon measure on $\mathbf{R}^{d}$ which only satisfies some polynomial growth

doi:10.5186/aasfm.2013.3809

2010 Mathematics Subject Classification: Primary 42B20; Secondary 42B25, 42B35, 42B30.

Key words: $\operatorname{RBMO}(\mu)$, Calderón-Zygmund operator, upper doubling measure, geometrically doubling, metric measure space.

The first author is supported by National Natural Science Foundation (Grant No. 10971228) of China, the second (corresponding) author is supported by National Natural Science Foundation (Grant No. 11071200) of China and the third author is supported by National Natural Science Foundation (Grant No. 11171027) of China and Program for Changjiang Scholars and Innovative Research Team in University of China.

*Corresponding author. 
condition: there exist positive constants $C$ and $n \in(0, d]$ such that for all $x \in \mathbf{R}^{d}$ and $r \in(0, \infty)$,

$$
\mu(B(x, r)) \leq C r^{n} .
$$

Such a measure needs not satisfy the doubling condition (1.1). Many results of the classical Calderón-Zygmund theory have been proved still valid if the doubling condition of the measure is replaced by (1.2); see, for example, [17, 18, 20, 21, 22] and their references.

Since the measures satisfying the polynomial growth condition (1.2) are different from, not more general than, the doubling measures, the Calderón-Zygmund theory associated with non-doubling measures is not in all respects a generalization of the corresponding theory on spaces of homogeneous type. To unite spaces of homogeneous type and Euclidean spaces with the underlying measure satisfying the polynomial growth condition (1.2), Hytönen [7] introduced a new class of metric measure spaces which satisfy the so-called upper doubling condition and the geometrically doubling condition (see Definitions 1.1 and 1.2 below).

Let $(\mathcal{X}, d, \mu)$ be a non-homogeneous space in the sense of Hytönen [7]. Hytönen [7] also introduced the space of regularized $\mathrm{BMO}$, namely, $\operatorname{RBMO}(\mu)$ (see Definition 1.3 below), which is a generalization of the regularized BMO of Tolsa [20] in the setting of $\mathbf{R}^{d}$ equipped with the measure satisfying (1.2). It has to be pointed out that the regularized BMO space introduced by Tolsa is a suitable substitute for the classical BMO space, which is small enough to fulfil the properties of the classical BMO space, such as the John-Nirenberg inequality, and large enough so that an $L^{2}(\mu)$ bounded Calderón-Zygmund operator is also bounded from $L^{\infty}(\mu)$ to $\operatorname{RBMO}(\mu)$. These properties are still true on non-homogeneous spaces in the sense of Hytönen; see [7, 1]. Recently, Lin and Yang [13] introduced the space of regularized BLO, which is a subspace of $\operatorname{RBMO}(\mu)$, and established several useful characterizations of this space.

The main purpose of this paper is to establish a new characterization of $\operatorname{RBMO}(\mu)$ in term of the John-Strömberg sharp maximal functions on the non-homogeneous spaces introduced by Hytönen [7]. As applications, we prove the equivalence between the $L^{p}(\mu)$-boundedness with $p \in(1, \infty)$ of the Calderón-Zygmund operator and several corresponding endpoint estimates. To state these results, we first recall some notions.

Definition 1.1. A metric measure space $(\mathcal{X}, d, \mu)$ is said to be upper doubling if $\mu$ is a Borel measure on $\mathcal{X}$ and there exist a dominating function $\lambda: \mathcal{X} \times(0, \infty) \rightarrow(0, \infty)$ and a positive constant $C_{\lambda}$ such that for each $x \in \mathcal{X}, r \rightarrow \lambda(x, r)$ is non-decreasing and, for all $x \in \mathcal{X}$ and $r \in(0, \infty)$,

$$
\mu(B(x, r)) \leq \lambda(x, r) \leq C_{\lambda} \lambda(x, r / 2) .
$$

Obviously, a space of homogeneous type is an upper doubling space if we choose $\lambda(x, r):=\mu(B(x, r))$. Also, $\left(\mathbf{R}^{d},|\cdot|, \mu\right)$ is also an upper doubling measure space if $\mu$ satisfies the polynomial growth condition (1.2).

The function $\lambda$ in Definition 1.1 needs not satisfy the additional property that there exists a positive constant $C$ such that for all $x, y \in \mathcal{X}$ with $d(x, y) \leq r$,

$$
\lambda(x, r) \leq C \lambda(y, r) .
$$


However, it was proved in [9] that there always exists another dominating function $\tilde{\lambda}$ such that $\tilde{\lambda} \leq \lambda, C_{\tilde{\lambda}} \leq C_{\lambda}$ and, for all $x, y \in \mathcal{X}$ with $d(x, y) \leq r, \widetilde{\lambda}(x, r) \leq C_{\tilde{\lambda}} \widetilde{\lambda}(y, r)$. Hence, in this paper, we always assume that the function $\lambda$ also satisfies (1.4).

Throughout the whole paper, we also always assume that the underlying metric space $(\mathcal{X}, d)$ satisfies the following geometrically doubling condition.

Definition 1.2. A metric space $(\mathcal{X}, d)$ is said to be geometrically doubling if there exists a positive integer $N_{0}$ such that for any ball $B(x, r) \subset \mathcal{X}$, there exists a finite number of balls covering $\left\{B\left(x_{i}, r / 2\right)\right\}_{i}$ of $B(x, r)$ such that the cardinality of this covering is at most $N_{0}$.

Some equivalent characterizations of the geometrically doubling property were proved in [7, Lemma 2.3]. Moreover, it is well known that spaces of homogeneous type are geometrically doubling spaces; see [2, p. 67]. In this paper, a metric measure space $(\mathcal{X}, d, \mu)$ is called a non-homogeneous metric measure space, if $\mu$ is upper doubling and $(\mathcal{X}, d)$ geometrically doubling.

We now recall the coefficients $\delta(B, S)$ for all balls $B$ and $S$ introduced in [7]. For all balls $B \subset S \subset \mathcal{X}$, let

$$
\delta(B, S):=\int_{(2 S) \backslash B} \frac{1}{\lambda\left(x_{B}, d\left(x, x_{B}\right)\right)} d \mu(x),
$$

where above and in what follows, for any ball $B:=B\left(x_{B}, r(B)\right)$ and $\rho \in(0, \infty)$, $\rho B:=B\left(x_{B}, \rho r(B)\right)$.

Definition 1.3. Let $\rho \in(1, \infty)$. A function $f \in L_{\text {loc }}^{1}(\mu)$ is said to be in the space $\operatorname{RBMO}(\mu)$ if there exist a positive constant $C$ and a number $f_{B}$ for any ball $B$ such that for all balls $B$,

$$
\frac{1}{\mu(\rho B)} \int_{B}\left|f(y)-f_{B}\right| d \mu(y) \leq C,
$$

and that for all balls $B \subset S$,

$$
\left|f_{B}-f_{S}\right| \leq C[1+\delta(B, S)]
$$

Moreover, the $\operatorname{RBMO}(\mu)$ norm of $f$ is defined to be the minimal constant $C$ as above and denoted by $\|f\|_{\operatorname{RBMO}(\mu)}$.

It was proved in $[7$, Lemma 4.6] that the space of $\operatorname{RBMO}(\mu)$ is independent of the choice of $\rho$.

The organization of this paper is as follows. In Section 2, we show that a $\mu$ measurable function $f$ belongs to $\operatorname{RBMO}(\mu)$ if and only if its John-Strömberg sharp maximal function is in $L^{\infty}(\mu)$ and the local integrability of $f$ is superfluous in the definition of $f \in \operatorname{RBMO}(\mu)$ (see Theorem 2.1 below). In Section 3, based on Corollary 2.1 in Section 2, we prove that for a Calderón-Zygmund operator, its boundedness on $L^{p}(\mu)$ with $p \in(1, \infty)$ is equivalent to its boundedness from Hardy space $H^{1}(\mu)$ (see Definition 3.1 in Section 3) into weak $L^{1}(\mu)$, or from $L^{\infty}(\mu)$ into $\operatorname{RBMO}(\mu)$ or some other estimates (see Theorem 3.1 below).

We remark that there exists other application of the new characterization of the space RBMO $(\mu)$ established in Section 2. Using Corollary 2.1 as an important tool, Lin and Yang [14] proved that a sublinear operator, which is bounded from the Hardy space $H^{1}(\mu)$ into $L^{1, \infty}(\mu)$ and from $L^{\infty}(\mu)$ into $\operatorname{RBMO}(\mu)$, is also bounded on $L^{p}(\mu)$ 
for all $p \in(1, \infty)$; see [14, Proposition 2.6] and the proofs of [14, Lemma 3.1 and Theorem 1.1] for more details.

Finally, we make some conventions on notation. Throughout this paper, we denote by $C$ a positive constant which is independent of the main parameters, but may vary from line to line. Constants with subscript, such as $C_{1}$, do not change in different occurrences. The symbol $f \lesssim g$ means that there exists a positive constant $C$ such that $f \leq C g$, and the symbol $f \sim g$ means $f \lesssim g \lesssim f$. For any ball $B \subset \mathcal{X}$, we denote its center and radius by $x_{B}$ and $r_{B}$, respectively. Also, for any subset $E \subset \mathcal{X}, \chi_{E}$ denotes its characteristic function.

\section{A new characterization of $\operatorname{RBMO}(\mu)$}

In this section, we introduce a space of functions via the John-Strömberg sharp maximal function and then prove that this space and $\operatorname{RBMO}(\mu)$ coincide with equivalent norms.

To introduce the John-Strömberg sharp maximal function, we first recall the notion of $(\alpha, \beta)$-doubling balls for $\alpha, \beta \in(1, \infty)$. Given $\alpha, \beta \in(1, \infty)$, a ball $B \subset \mathcal{X}$ is called $(\alpha, \beta)$-doubling if $\mu(\alpha B) \leq \beta \mu(B)$. It was proved in [7, Lemmas 3.2 and 3.3] that for any $\alpha \in(1, \infty), \beta \in\left(C_{\lambda}^{\log _{2} \alpha}, \infty\right)$ and any ball $B \subset \mathcal{X}$, there exists some $j \in \mathbf{N}:=\{1,2, \cdots\}$ such that $\alpha^{j} B$ is $(\alpha, \beta)$-doubling. On the other hand, let $(\mathcal{X}, d)$ be geometrically doubling, $\beta \in\left(\alpha^{n}, \infty\right)$ with $n:=\log _{2} N_{0}$ and $\mu$ a Borel measure on $\mathcal{X}$ which is finite on bounded sets, where $N_{0}$ is as in Definition 1.2. Then for $\mu$-almost every $x \in \mathcal{X}$, there exist arbitrarily small $(\alpha, \beta)$-doubling balls centered at $x$; furthermore, the radii of these balls may be chosen to be of the form $\alpha^{-j} r$ for $j \in \mathbf{N}$ and any preassigned number $r \in(0, \infty)$. In this section, for fixed $\varrho \in[1, \infty)$, by a doubling ball $B$, we always mean that $B$ is a $\left(6 \varrho^{2}, \beta_{6 \varrho^{2}}\right)$-doubling ball with $\beta_{6 \varrho^{2}}:=\left(6 \varrho^{2}\right)^{\max \{n, d\}}+30^{n}+30^{d}$, where $d$ satisfies $C_{\lambda}^{\log _{2}\left(6 \varrho^{2}\right)}=\left(6 \varrho^{2}\right)^{d}$. For any ball $B \subset \mathcal{X}$, we use the symbol $\widetilde{B}$ to denote the smallest $\left(6 \varrho^{2}, \beta_{6 \varrho^{2}}\right)$-doubling ball of the form $\left(6 \varrho^{2}\right)^{j} B$ with $j \in \mathbf{Z}_{+}:=\{0,1, \cdots\}$.

Let $f$ be a $\mu$-measurable function. If $f$ is real-valued, then for all balls $B$ with $\mu(B) \neq 0$, define the median value of $f$ on the ball $B$, denoted by $m_{f}(B)$, to be one of the numbers such that

$$
\mu\left(\left\{x \in B: f(x)>m_{f}(B)\right\}\right) \leq \mu(B) / 2
$$

and

$$
\mu\left(\left\{x \in B: f(x)<m_{f}(B)\right\}\right) \leq \mu(B) / 2 ;
$$

see [6, p. 161]. For all balls $B$ with $\mu(B)=0$, set $m_{f}(B)=0$. If $f$ is complex-valued, we take $m_{f}(B):=m_{\operatorname{Re} f}(B)+i m_{\operatorname{Im} f}(B)$.

Let $s \in(0,1)$ and $\varrho \in(1, \infty)$. For any fixed ball $B$ and $\mu$-measurable function $f$, define $m_{0, s ; B}^{\varrho}(f)$ by setting

$$
m_{0, s ; B}^{\varrho}(f):=\inf \{t>0: \mu(\{y \in B:|f(y)|>t\})<s \mu(\varrho B)\}
$$

when $\mu(B)>0$, and $m_{0, s ; B}^{\varrho}(f):=0$ when $\mu(B)=0$. For any $\mu$-measurable function $f$, the John-Strömberg sharp maximal function $M_{0, s}^{\varrho, \sharp}(f)$ is defined by setting, for all $x \in \mathcal{X}$,

$$
M_{0, s}^{\rho, \sharp}(f)(x):=\sup _{B \ni x} m_{0, s ; B}^{\varrho}\left(f-m_{f}(\widetilde{B})\right)+\sup _{\substack{x \in B \subset S \\ B, S \\\left(6 \varrho^{2}, \beta \\\left(\varrho^{2}\right)\right. \text {-doubling }}} \frac{\left|m_{f}(B)-m_{f}(S)\right|}{1+\delta(B, S)} .
$$


Using $M_{0, s}^{\varrho, \sharp}$, we introduce the space $\operatorname{RBMO}_{0, s}(\mu)$ as follows.

Definition 2.1. Let $s \in(0,1)$ and $\varrho \in(1, \infty)$. A $\mu$-measurable function $f$ is said to belong to the space $\operatorname{RBMO}_{0, s}(\mu)$ if $M_{0, s}^{\varrho, \sharp}(f) \in L^{\infty}(\mu)$. Moreover, $\left\|M_{0, s}^{\varrho, \sharp}(f)\right\|_{L^{\infty}(\mu)}$ is defined to be the $\mathrm{RBMO}_{0, s}(\mu)$ norm of $f$ and denoted by $\|f\|_{\mathrm{RBMO}_{0, s}(\mu)}$.

The main result of this section is as follows.

Theorem 2.1. Let $\varrho \in(1, \infty)$ and $s \in\left(0, \beta_{6 \varrho^{2}}^{-2} / 4\right)$. Then the spaces $\operatorname{RBMO}(\mu)$ and $\mathrm{RBMO}_{0, s}(\mu)$ coincide with equivalent norms.

If $(\mathcal{X}, d, \mu):=\left(\mathbf{R}^{d},|\cdot|, d x\right)$, Strömberg [19] proved that $\operatorname{RBMO}(\mu)=\operatorname{RBMO}_{0, s}(\mu)$ if and only if $s \in(0,1 / 2]$. Moreover, if $\mu$ is an absolutely continuous measure on $\mathbf{R}^{d}$, namely, there exists a weight $w$ such that $d \mu=w d x$, Lerner [12] established the JohnStrömberg characterization of $\operatorname{BMO}(w)$. Furthermore, if $(\mathcal{X}, d, \mu):=\left(\mathbf{R}^{d},|\cdot|, \mu\right)$ with $\mu$ satisfying (1.2), it was proved that $\operatorname{RBMO}(\mu)=\operatorname{RBMO}_{0, s}(\mu)$ for $s \in\left(0, \beta_{d}^{-2} / 2\right)$ with $\beta_{d} \in\left(2^{d}, \infty\right)$ in [6].

By Theorem 2.1, we obtain the following conclusion.

Corollary 2.1. Let $\varrho \in(1, \infty)$ and $\varphi$ be a strictly increasing and nonnegative continuous function on $[0, \infty)$ such that $\lim _{t \rightarrow \infty} \varphi(t)=\infty$. If $f \in L_{\mathrm{loc}}^{1}(\mu)$ and there exists a positive constant $C$ such that for all balls $B \subset \mathcal{X}$,

$$
\frac{1}{\mu(\varrho B)} \int_{B} \varphi\left(\left|f(x)-m_{f}(\widetilde{B})\right|\right) d \mu(x) \leq C
$$

and that for all $\left(6 \varrho^{2}, \beta_{6 \varrho^{2}}\right)$-doubling balls $B \subset S,\left|m_{f}(B)-m_{f}(S)\right| \leq C[1+\delta(B, S)]$, then $f \in \operatorname{RBMO}(\mu)$.

A typical example of $\varphi$ satisfying Corollary 2.1 is $\varphi(r):=r^{p}$ for all $r \in[0, \infty)$ with $p \in(0, \infty)$. We remark that if $p \in[1, \infty)$, the conclusion that a $\mu$-locally integrable function satisfying the hypothesis of Corollary 2.1 belongs to $\operatorname{RBMO}(\mu)$ can be deduced from the John-Nirenberg inequality established by Hytönen in [7]. However, if $p \in(0,1)$, this conclusion cannot be deduced from the John-Nirenberg inequality anymore. Other typical examples of $\varphi$ satisfying the hypothesis of Corollary 2.1 are

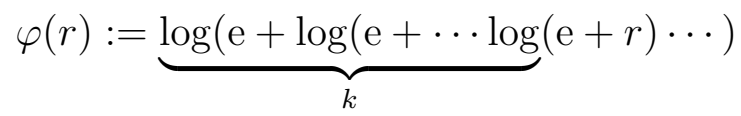

with $k \in \mathbf{N}$.

The remaining part of this section is devoted to the proofs of Theorem 2.1 and Corollary 2.1. To this end, we first establish the corresponding John-Nirenberg inequality for the space $\operatorname{RBMO}_{0, s}(\mu)$ with $\varrho \in(1, \infty)$ and $s \in\left(0, \beta_{6 \varrho^{2}}^{-2} / 4\right)$. It plays an important role in the proof of Theorem 2.1.

Proposition 2.1. For any $\varrho \in(1, \infty)$ and $s \in\left(0, \beta_{6 \varrho^{2}}^{-2} / 4\right)$, there exist two positive constant $C_{\varrho}$ and $c_{\varrho}$ such that for all $f \in \mathrm{RBMO}_{0, s}(\mu)$, all balls $B_{0} \subset \mathcal{X}$ and $t \in(0, \infty)$,

$$
\mu\left(\left\{x \in B_{0}:\left|f(x)-m_{f}\left(\widetilde{B_{0}}\right)\right|>t\right\}\right) \leq C_{\varrho} \mathrm{e}^{-\frac{c_{\varrho} t}{\|f\|_{\mathrm{RBMO}_{0, s}(\mu)}}} \mu\left(\varrho^{2} B_{0}\right) .
$$

We point out that Proposition 2.1 is a generalization of the John-Nirenberg inequality for the space $\operatorname{RBMO}(\mu)$ on the non-homogeneous spaces, which was proved in [7, Proposition 6.1] by Hytönen. 
To prove Proposition 2.1, we need some technical lemmas. The following Lemma 2.1 is a direct corollary of [3, Theorem 1.2] and [7, Lemma 2.5].

Lemma 2.1. Let $(\mathcal{X}, d)$ be a geometrically doubling metric space. Then every family $\mathcal{F}$ of balls of uniformly bounded diameter contains an at most countable disjointed subfamily $\mathcal{G}$ such that $\cup_{B \in \mathcal{F}} B \subset \cup_{B \in \mathcal{G}} 5 B$.

Lemma 2.2. [9, Lemma 2.1]

(i) For all balls $B \subset S \subset R, \delta(B, S) \leq \delta(B, R)$.

(ii) For any $\rho \in[1, \infty)$, there exists a positive constant $C$, depending on $\rho$, such that for all balls $B \subset S$ with $r(S) \leq \rho r(B), \delta(B, S) \leq C$.

(iii) There exists a positive constant $\widetilde{C}$ such that for all balls $B, \delta(B, \widetilde{B}) \leq C$.

(iv) There exists a positive constant $c$ such that for all balls $B \subset R \subset S, \delta(B, S) \leq$ $\delta(B, R)+c \delta(R, S)$. In particular, if $B$ and $R$ are concentric, then $c=1$.

(v) There exists a positive constant $\widetilde{c}$ such that for all balls $B \subset R \subset S, \delta(R, S) \leq$ $\widetilde{c}[1+\delta(B, S)]$; moreover, if $B$ and $R$ are concentric, then $\delta(R, S) \leq \delta(B, S)$.

Lemma 2.3. [7, Corollary 3.6] Let $(\mathcal{X}, d)$ be a geometrically doubling metric space and $\mu$ a Borel measure on $\mathcal{X}$ which is finite on bounded sets. Let $\beta \in\left(5^{n}, \infty\right)$. Then for all $f \in L_{\mathrm{loc}}^{1}(\mu)$ and $\mu$-almost every $x \in \mathcal{X}$,

$$
f(x)=\lim _{\substack{B \downarrow x \\(5, \beta)-\text { doubling }}} \frac{1}{\mu(B)} \int_{B} f(y) d \mu(y) .
$$

Let $\varrho \in(1, \infty)$. The doubling maximal operator $N$ and the doubling local maximal operator $M_{0, s}^{d}$ are, respectively, defined by setting, for all $f \in L_{\text {loc }}^{1}(\mu)$ and $x \in \mathcal{X}$,

$$
N(f)(x):=\sup _{B\left(6 \varrho^{2}, \beta_{\left.6 \varrho^{2}\right)- \text { doubling }}\right.} \frac{1}{\mu(B)} \int_{B}|f(y)| d \mu(y)
$$

and, for all $\mu$-measurable functions $f$ and $x \in \mathcal{X}$,

$$
M_{0, s}^{d}(f)(x):=\sup _{B\left(6 \varrho^{2}, \beta_{\left.6 \varrho^{2}\right)}\right) \text {-doubling }} m_{0, s ; B}^{\varrho}(f) .
$$

Lemma 2.4. Let $\varrho \in(1, \infty)$ and $s \in\left(0, \beta_{6 \varrho^{2}}^{-1}\right)$. If $f$ is a $\mu$-measurable function, then for all $t \in(0, \infty)$,

$$
\mu(\{x \in \mathcal{X}:|f(x)|>t\}) \leq \mu\left(\left\{x \in \mathcal{X}: M_{0, s}^{d}(f)(x) \geq t\right\}\right) .
$$

Proof. It is easy to see that for all $t \in(0, \infty)$,

$$
\{x \in \mathcal{X}:|f(x)|>t\}=\left\{x \in \mathcal{X}: \chi_{\{y \in \mathcal{X}:|f(y)|>t\}}(x)=1\right\}
$$

which, along with Lemma 2.3 and the fact $s \in\left(0, \beta_{6 \varrho^{2}}^{-1}\right)$, implies that for $\mu$-almost every $x \in \mathcal{X}$ satisfying $|f(x)|>t$,

$$
N\left(\chi_{\{y \in \mathcal{X}:|f(y)|>t\}}\right)(x) \geq \chi_{\{y \in \mathcal{X}:|f(y)|>t\}}(x)=1>s \beta_{6 \varrho^{2}} .
$$

This means that

$$
\{x \in \mathcal{X}:|f(x)|>t\} \subset\left\{x \in \mathcal{X}: N\left(\chi_{\{y \in \mathcal{X}:|f(y)|>t\}}\right)(x)>\beta_{6 \varrho^{2}} s\right\} \cup \Theta,
$$

where $\mu(\Theta)=0$. By Lemma 2.3 again, we see that for any $x \in \mathcal{X}$ satisfying

$$
N\left(\chi_{\{y \in \mathcal{X}:|f(y)|>t\}}\right)(x)>\beta_{6 \varrho^{2}} s,
$$


there exists a $\left(6 \varrho^{2}, \beta_{6 \varrho^{2}}\right)$-doubling ball $B$ containing $x$ such that

$$
\frac{1}{\mu(B)} \int_{B} \chi_{\{y \in \mathcal{X}:|f(y)|>t\}}(y) d \mu(y)>s \beta_{6 \varrho^{2}} .
$$

This means that

$$
\mu(\{y \in B:|f(y)|>t\})>s \beta_{6 \varrho^{2}} \mu(B) .
$$

Notice that

$$
\mu(\varrho B) \leq \mu\left(6 \varrho^{2} B\right) \leq \beta_{6 \varrho^{2}} \mu(B) .
$$

Hence,

$$
\mu(\{y \in B:|f(y)|>t\})>s \mu(\varrho B) .
$$

On the other hand, by the definition of $m_{0, s: B}^{\varrho}(f)$, we easily conclude that for any $r \in\left(m_{0, s: B}^{\varrho}(f), \infty\right), \mu(\{y \in B:|f(y)|>r\})<s \mu(\varrho B)$. Therefore, $m_{0, s ; B}^{\varrho}(f) \geq t$ and hence $M_{0, s}^{d}(f)(x) \geq t$, which implies that

$$
\{x \in \mathcal{X}:|f(x)|>t\} \subset\left\{x \in \mathcal{X}: M_{0, s}^{d}(f)(x) \geq t\right\} \cup \Theta .
$$

The desired conclusion (2.1) then follows directly, which completes the proof of Lemma 2.4.

Lemma 2.5. Let $\varrho \in(1, \infty), s \in\left(0, \beta_{6 \varrho^{2}}^{-1} / 2\right]$ and $B$ be a $\left(6 \varrho^{2}, \beta_{6 \varrho^{2}}\right)$-doubling ball. Then for all $\mu$-measurable real-valued functions $f$,

$$
\left|m_{f}(B)\right| \leq m_{0, s ; B}^{\varrho}(f) .
$$

The proof of Lemma 2.5 is similar to that of [6, Lemma 1]. For brevity, we omit the details here. Moreover, by Lemma 2.5, we easily conclude that for all $\mu$-measurable complex-valued functions $f$ and all $\left(6 \varrho^{2}, \beta_{6 \varrho^{2}}\right)$-doubling balls $B \subset \mathcal{X}$,

$$
\left|m_{f}(B)\right| \leq\left|m_{\operatorname{Re} f}(B)\right|+\left|m_{\operatorname{Im} f}(B)\right| \leq m_{0, s ; B}^{\varrho}(\operatorname{Re} f)+m_{0, s ; B}^{\varrho}(\operatorname{Im} f) \leq 2 m_{0, s ; B}^{\varrho}(f),
$$

which is used in Section 3.

Lemma 2.6. Let $\rho \in(1, \infty)$ and $\varrho \in[1, \infty)$. For any given $f \in L_{\text {loc }}^{1}(\mu)$, let $\|f\|_{*}$ be the minimal nonnegative constant $C$ such that for all balls $B$,

$$
\frac{1}{\mu(\rho B)} \int_{B}\left|f(y)-m_{\widetilde{B}}(f)\right| d \mu(y) \leq C,
$$

and that for all $\left(6 \varrho^{2}, \beta_{6 \varrho^{2}}\right)$-doubling balls $B \subset S$,

$$
\left|m_{B}(f)-m_{S}(f)\right| \leq C[1+\delta(B, S)],
$$

where $\widetilde{B}$ denotes the smallest $\left(6 \varrho^{2}, \beta_{6 \varrho^{2}}\right)$-doubling ball with the form $\left(6 \varrho^{2}\right)^{i} B$ for $i \in \mathbf{Z}_{+}$, and for any $g \in L_{\text {loc }}^{1}(\mu)$ and any ball $B \subset \mathcal{X}, m_{B}(g)$ denotes the mean of $g$ over $B$, namely, $m_{B}(g):=\frac{1}{\mu(B)} \int_{B} g(x) d \mu(x)$. Then $\|\cdot\|_{*}$ is a norm of $\operatorname{RBMO}(\mu)$, which is equivalent to $\|\cdot\|_{\mathrm{RBMO}(\mu)}$.

Lemma 2.6 is a variant of [9, Proposition 2.2], in which $\varrho=1$. The proof therein is still valid in the current case.

Proof of Proposition 2.1. We use some ideas from Hytönen [7] and adapt them to the space $\mathrm{RBMO}_{0, s}(\mu)$. It suffices to prove that there exist two positive constant 
$\widetilde{C}_{\varrho}$ and $\widetilde{c}_{\varrho}$ such that for any real-value function $f \in \mathrm{RBMO}_{0, s}(\mu)$, any ball $B_{0} \subset \mathcal{X}$ and $t \in(0, \infty)$,

$$
\mu\left(\left\{x \in B_{0}:\left|f(x)-m_{f}\left(\widetilde{B_{0}}\right)\right|>t\right\}\right) \leq \widetilde{C}_{\varrho} e^{-\frac{\tilde{c}_{e} t}{\|f\|_{\mathrm{RBMO}_{0, s}(\mu)}}} \mu\left(\varrho^{2} B_{0}\right) .
$$

In fact, for any complex-valued function $f$, we write $f:=f_{1}+i f_{2}$, where $f_{1}$ and $f_{2}$ are, respectively, the real and the imaginary parts of $f$. Notice that $\left\|f_{1}\right\|_{\mathrm{RBMO}_{0, s}(\mu)}$ and $\left\|f_{2}\right\|_{\mathrm{RBMO}_{0, s}(\mu)}$ are both not greater than $\|f\|_{\mathrm{RBMO}_{0, s}(\mu)}$. Therefore, if the inequality (2.3) holds for the real-valued functions $f_{1}$ and $f_{2}$, then

$$
\begin{aligned}
& \mu\left(\left\{x \in B_{0}:\left|f(x)-m_{f}\left(\widetilde{B_{0}}\right)\right|>t\right\}\right) \\
& \leq \mu\left(\left\{x \in B_{0}:\left|f_{1}(x)-m_{f_{1}}\left(\widetilde{B_{0}}\right)\right|>t / 2\right\}\right) \\
& +\mu\left(\left\{x \in B_{0}:\left|f_{2}(x)-m_{f_{2}}\left(\widetilde{B_{0}}\right)\right|>t / 2\right\}\right) \\
& \leq \widetilde{C}_{\varrho}\left[e^{-\frac{\tilde{c}_{Q} t}{2\left\|f_{1}\right\|_{\mathrm{RBMO}_{0, s}(\mu)}}}+e^{-\frac{\tilde{c}_{Q} t}{2\left\|f_{2}\right\|_{\mathrm{RBMO}_{0, s}(\mu)}}}\right] \mu\left(\varrho^{2} B_{0}\right) \\
& \leq \widetilde{C}_{\varrho}\left[e^{-\frac{\tilde{c}_{Q} t}{2\|f\|_{\mathrm{RBO}_{0, s}(\mu)}}}+e^{-\frac{\tilde{c}_{Q} t}{2\|f\|_{\mathrm{RBO}_{0, s}(\mu)}}}\right] \mu\left(\varrho^{2} B_{0}\right) \\
& \leq 2 \widetilde{C}_{\varrho} e^{-\frac{\tilde{c}_{\varrho} t}{2\|f\|_{\mathrm{RBMO}_{0, s}(\mu)}}} \mu\left(\varrho^{2} B_{0}\right) .
\end{aligned}
$$

Therefore, Proposition 2.1 holds for all complex-valued functions with $C_{\varrho}:=2 \widetilde{C}_{\varrho}$ and $c_{\varrho}:=\widetilde{c}_{\varrho} / 2$.

To show (2.3), without loss of generality, we may assume that $\|f\|_{\mathrm{RBMO}_{0, s}(\mu)}>$ 0. Otherwise, by the definition of $\|f\|_{\mathrm{RBMO}_{0, s}(\mu)}$, we easily conclude that for all $\left(6 \varrho^{2}, \beta_{6 \varrho^{2}}\right)$-doubling balls $B \subset S, m_{f}(B)=m_{f}(S)$ and

$$
\sup _{B \subset \mathcal{X}} m_{0, s ; B}^{\varrho}\left(f-m_{f}(\widetilde{B})\right)=0 .
$$

Thus, there exists a constant $M$ such that for any $\left(6 \varrho^{2}, \beta_{6 \varrho^{2}}\right)$-doubling ball $B, m_{f}(B)$ $=M$ and hence $m_{0, s ; B}(f-M)=0$. This further implies that for all $x \in \mathcal{X}$, $M_{0, s}^{d}(f-M)(x)=0$. From this and Lemma 2.4, it follows that $f(x)=M$ for $\mu$-almost every $x \in \mathcal{X}$, which implies that for any ball $B_{0} \subset \mathcal{X}$ and $t \in(0, \infty)$,

$$
\mu\left(\left\{x \in B_{0}:\left|f(x)-m_{f}\left(\widetilde{B_{0}}\right)\right|>t\right\}\right)=0 .
$$

Therefore, the inequality (2.3) holds in this case.

Denote by $L$ a large positive constant which is determined later. Choose $\gamma \in$ $\left(2 \beta_{6 \varrho^{2}}, \infty\right)$ such that $\gamma s<\beta_{6 \varrho^{2}}^{-1} / 2$. It is easy to see that for all $x \in B_{0}$ satisfying $\left|f(x)-m_{f}\left(\widetilde{B_{0}}\right)\right|>2 L$,

$$
\chi_{\left\{y \in B_{0}:\left|f(y)-m_{f}\left(\widetilde{B_{0}}\right)\right|>2 L\right\}}(x)=1>\gamma s .
$$

On the other hand, from Lemma 2.3, it follows that for $\mu$-almost every $x \in B_{0}$ satisfying $\left|f(x)-m_{f}\left(\widetilde{B_{0}}\right)\right|>2 L$,

$$
\chi_{\left\{y \in B_{0}:\left|f(y)-m_{f}\left(\widetilde{B_{0}}\right)\right|>2 L\right\}}(x)=\lim _{\substack{B \downarrow x \\\left(6 \varrho^{2}, \beta_{6 \varrho^{2}}\right) \text {-doubling }}} \frac{1}{\mu(B)} \int_{B} \chi_{\left\{y \in B_{0}:\left|f(y)-m_{f}\left(\widetilde{B_{0}}\right)\right|>2 L\right\}}(y) d \mu(y) .
$$


A new characterization of regularized BMO spaces on non-homogeneous spaces and its applications 11

Therefore, for $\mu$-almost every $x \in B_{0}$ satisfying $\left|f(x)-m_{f}\left(\widetilde{B_{0}}\right)\right|>2 L$, there exists an arbitrarily small $\left(6 \varrho^{2}, \beta_{6} \varrho^{2}\right)$-doubling ball $B:=B\left(x,\left(6 \varrho^{2}\right)^{-k} r\right)$ such that

$$
\frac{1}{\mu(B)} \int_{B} \chi_{\left\{y \in \mathcal{X}:\left|f(y)-m_{f}\left(\widetilde{B_{0}}\right)\right|>2 L\right\}}(y) d \mu(y)>\gamma s .
$$

This means that

$$
\mu\left(\left\{y \in B:\left|f(y)-m_{f}\left(\widetilde{B_{0}}\right)\right|>2 L\right\}\right)>\gamma s \mu(B) .
$$

Let $B_{x}^{*}$ be the maximal $\left(6 \varrho^{2}, \beta_{6 \varrho^{2}}\right)$-doubling ball of the form $B\left(x,\left(6 \varrho^{2}\right)^{-k} r\right)$ with $k \in \mathbf{N}$ satisfying $B_{x}^{*} \subset \varrho B_{0}$ and $(2.4)$.

Denote by $B_{x}^{* *}$ the smallest $\left(6 \varrho^{2}, \beta_{6 \varrho^{2}}\right)$-doubling ball of the form $\left(6 \varrho^{2}\right)^{k} B_{x}^{*}$ with $k \in \mathbf{N}$. We claim that

$$
\left|m_{f}\left(B_{x}^{* *}\right)-m_{f}\left(\widetilde{B_{0}}\right)\right| \leq 2 L .
$$

To show (2.5), we consider the following three cases.

Case $A . B_{x}^{* *} \nsubseteq \varrho B_{0}$ and $r\left(B_{x}^{* *}\right) \leq r\left(\widetilde{B_{0}}\right)$. In this case, $B_{x}^{* *} \subset 6 \varrho^{2} \widetilde{B_{0}}$. By Definition 2.1, we conclude that

$$
\left|m_{f}\left(\widetilde{B_{0}}\right)-m_{f}\left(\widetilde{6 \varrho^{2} \widetilde{B_{0}}}\right)\right| \leq\left[1+\delta\left(\widetilde{B_{0}}, \widetilde{6 \varrho^{2} \widetilde{B_{0}}}\right)\right]\|f\|_{\mathrm{RBMO}_{0, s}(\mu)} .
$$

On the other hand,

$$
\left|m_{f}\left(B_{x}^{* *}\right)-m_{f}\left(\widetilde{6 \varrho^{2} \widetilde{B_{0}}}\right)\right| \leq\left[1+\delta\left(B_{x}^{* *}, \widetilde{6 \varrho^{2} \widetilde{B_{0}}}\right)\right]\|f\|_{\mathrm{RBMO}_{0, s}(\mu)} .
$$

Let $\left(6 \varrho^{2}\right)^{k_{0}} B_{x}^{*}$ be the smallest expansion of $B_{x}^{*}$ such that $\left(6 \varrho^{2}\right)^{k} B_{x}^{*} \nsubseteq \subseteq B_{0}$ with $k \in \mathbf{N}$. Then, $r\left(\left(6 \varrho^{2}\right)^{k_{0}} B_{x}^{*}\right) \sim r\left(B_{0}\right),\left(6 \varrho^{2}\right)^{k_{0}-1} B_{x}^{*} \subset \varrho B_{0}$ and $\left(6 \varrho^{2}\right)^{k_{0}-1} B_{x}^{*} \subset B_{x}^{* *}$. Thus, by (iv), (v), (ii) and (iii) of Lemma 2.2, we see that there exists a positive constant $C_{1,1}$, depending on $\varrho$ and $\mu$, such that

$$
\begin{aligned}
& 2+\delta\left(\widetilde{B_{0}}, \widetilde{6 \varrho^{2} \widetilde{B_{0}}}\right)+\delta\left(B_{x}^{* *}, \widetilde{6 \varrho^{2} \widetilde{B_{0}}}\right) \\
& \leq 2+\delta\left(\widetilde{B_{0}}, 6 \varrho^{2} \widetilde{B_{0}}\right)+\delta\left(6 \varrho^{2} \widetilde{B_{0}}, \widetilde{6 \varrho^{2} \widetilde{B_{0}}}\right) \\
& +\delta\left(\left(6 \varrho^{2}\right)^{k_{0}-1} B_{x}^{*}, 6 \varrho^{2} B_{0}\right)+c\left[\delta\left(6 \varrho^{2} B_{0}, 6 \varrho^{2} \widetilde{B_{0}}\right)+\delta\left(6 \varrho^{2} \widetilde{B_{0}}, \widetilde{6 \varrho^{2}} \widetilde{B_{0}}\right)\right] \\
& \leq C_{1,1} \text {. }
\end{aligned}
$$

From this, the estimates (2.6) and (2.7), it follows that

$$
\begin{aligned}
\left|m_{f}\left(B_{x}^{* *}\right)-m_{f}\left(\widetilde{B_{0}}\right)\right| & \leq\left|m_{f}\left(B_{x}^{* *}\right)-m_{f}\left(\widetilde{6 \varrho^{2} \widetilde{B_{0}}}\right)\right|+\left|m_{f}\left(\widetilde{6 \varrho^{2} \widetilde{B_{0}}}\right)-m_{f}\left(\widetilde{B_{0}}\right)\right| \\
& \leq C_{1,1}\|f\|_{\mathrm{RBMO}_{0, s}(\mu)} .
\end{aligned}
$$

Case B. $B_{x}^{* *} \nsubseteq \varrho B_{0}$ and $r\left(B_{x}^{* *}\right)>r\left(\widetilde{B_{0}}\right)$. In this case, $\widetilde{B_{0}} \subset 6 \varrho^{2} B_{x}^{* *}$. It follows, from Definition 2.1, that

$$
\left|m_{f}\left(B_{x}^{* *}\right)-m_{f}\left(\widetilde{\left(6 \varrho^{2}\right)^{2} B_{x}^{* *}}\right)\right| \leq\left[1+\delta\left(B_{x}^{* *}, \widetilde{\left(6 \varrho^{2}\right)^{2} B_{x}^{* *}}\right)\right]\|f\|_{\operatorname{RBMO}_{0, s}(\mu)}
$$


and

$$
\left|m_{f}\left(\widetilde{B_{0}}\right)-m_{f}\left(\widetilde{\left(6 \varrho^{2}\right)^{2} B_{x}^{* *}}\right)\right| \leq\left[1+\delta\left(\widetilde{B_{0}}, \widetilde{\left(6 \varrho^{2}\right)^{2} B_{x}^{* *}}\right)\right]\|f\|_{\operatorname{RBMO}_{0, s}(\mu)} .
$$

Since $\widetilde{B_{0}} \subset 6 \varrho^{2} B_{x}^{* *}$, it follows that there exists a positive constant $m \in \mathbf{N}$ such that $r\left(\widetilde{B_{0}}\right) \geq r\left(\left(6 \varrho^{2}\right)^{m} B_{x}^{*}\right) /\left(6 \varrho^{2}\right)^{2}$ and $\widetilde{B_{0}} \subset\left(6 \varrho^{2}\right)^{m} B_{x}^{*} \subset\left(\widetilde{\left.6 \varrho^{2}\right)^{2} B_{x}^{* *}}\right.$. Thus, $r\left(\widetilde{B_{0}}\right) \sim$ $r\left(\left(6 \varrho^{2}\right)^{m} B_{x}^{*}\right)$ and hence, by (iv), (v), (ii) and (iii) of Lemma 2.2, we see that there exists a positive constant $C_{1,2}$, depending on $\varrho$ and $\mu$, such that

$$
\begin{aligned}
& 2+\delta\left(B_{x}^{* *}, \widetilde{\left(6 \varrho^{2}\right)^{2} B_{x}^{* *}}\right)+\delta\left(\widetilde{B_{0}},\left(\widetilde{\left.6 \varrho^{2}\right)^{2} B_{x}^{* *}}\right)\right. \\
& \leq 3+\delta\left(B_{x}^{* *},\left(6 \varrho^{2}\right)^{2} B_{x}^{* *}\right)+\delta\left(\left(6 \varrho^{2}\right)^{2} B_{x}^{* *}, \widetilde{\left(6 \varrho^{2}\right)^{2} B_{x}^{* *}}\right) \\
& +c\left[\delta\left(\widetilde{B_{0}},\left(6 \varrho^{2}\right)^{m} B_{x}^{*}\right)+\delta\left(B_{x}^{*},\left(6 \varrho^{2}\right)^{2} B_{x}^{* *}\right)+\delta\left(\left(6 \varrho^{2}\right)^{2} B_{x}^{* *},\left(\widetilde{\left.6 \varrho^{2}\right)^{2} B_{x}^{* *}}\right)\right]\right. \\
& \leq C_{1,2} \text {. }
\end{aligned}
$$

This, along with (2.8) and (2.9), implies that

$$
\begin{aligned}
\left|m_{f}\left(B_{x}^{* *}\right)-m_{f}\left(\widetilde{B_{0}}\right)\right| \leq & \left|m_{f}\left(B_{x}^{* *}\right)-m_{f}\left(\widetilde{\left(6 \varrho^{2}\right)^{2} B_{x}^{* *}}\right)\right| \\
& +\left|m_{f}\left(\widetilde{\left(6 \varrho^{2}\right)^{2} B_{x}^{* *}}\right)-m_{f}\left(\widetilde{B_{0}}\right)\right| \leq C_{1,2}\|f\|_{\mathrm{RBMO}_{0, s}(\mu)} .
\end{aligned}
$$

Case C. $B_{x}^{* *} \subseteq \varrho B_{0}$. Recall the fact $\gamma s<\beta_{6 \varrho^{2}}^{-1} / 2$. Then we choose $\eta \in(0, \infty)$ such that $\gamma s+\eta<\beta_{6 \varrho^{2}}^{-1} / 2$. From the choice of $B_{x}^{*}$, it follows that (2.4) does not hold for the ball $B_{x}^{* *}$, that is,

$$
\mu\left(\left\{y \in B_{x}^{* *}:\left|f(y)-m_{f}\left(\widetilde{B_{0}}\right)\right|>2 L\right\}\right) \leq \gamma s \mu\left(B_{x}^{* *}\right)<(\gamma s+\eta) \mu\left(B_{x}^{* *}\right) .
$$

This means that

$$
m_{0, \gamma s+\eta ; B_{x}^{* *}}^{\varrho}\left(f-m_{f}\left(\widetilde{B_{0}}\right)\right) \leq 2 L,
$$

which, along with Lemma 2.5, implies that

$$
\left|m_{f}\left(B_{x}^{* *}\right)-m_{f}\left(\widetilde{B_{0}}\right)\right|=\left|m_{f-m_{f}\left(\widetilde{B_{0}}\right)}\left(B^{* *}\right)\right| \leq m_{0, \gamma s+\eta ; B_{x}^{* *}}^{\varrho}\left(f-m_{f}\left(\widetilde{B_{0}}\right)\right) \leq 2 L,
$$

where we used the fact that for any ball $B, c \in \mathbf{C}$ and $\mu$-measurable function $h$, $m_{h}(B)-c=m_{h-c}(B)$.

Let $C_{1}:=\max \left\{C_{1,1}, C_{1,2}\right\}$. Choose $L \geq \frac{C_{1}}{2}\|f\|_{\mathrm{RBMO}_{0, s}(\mu)}$. Then (2.5) is true.

Let $C_{2} \in(1, \infty)$ be a constant, depending on $\varrho$ and $\mu$, such that $1+\delta\left(B_{x}^{*}, B_{x}^{* *}\right) \leq$ $C_{2}$. Then, if $L \geq 2 C_{2}\|f\|_{\mathrm{RBMO}_{0, s}(\mu)}$, by Definition 2.1, (2.5) and Lemma 2.2(iii), we conclude that

$$
\begin{aligned}
& \left|m_{f}\left(B_{x}^{*}\right)-m_{f}\left(\widetilde{B_{0}}\right)\right| \\
& \leq\left|m_{f}\left(B_{x}^{*}\right)-m_{f}\left(B_{x}^{* *}\right)\right|+\left|m_{f}\left(B_{x}^{* *}\right)-m_{f}\left(\widetilde{B_{0}}\right)\right| \\
& \leq\left[1+\delta\left(B_{x}^{*}, B_{x}^{* *}\right)\right]\|f\|_{\mathrm{RBMO}_{0, s}(\mu)}+2 L \leq C_{2}\|f\|_{\mathrm{RBMO}_{0, s}(\mu)}+2 L \leq \frac{5}{2} L .
\end{aligned}
$$

By Lemma 2.1, we choose disjoint balls $\left\{B_{x_{i}}^{*}\right\}_{i}$ among the balls $\left\{B_{x}^{*}\right\}_{x \in B_{0}}$ so that the expanded balls $\left\{5 B_{x_{i}}^{*}\right\}_{i}$ cover all the original $B_{x}^{*}$. It follows, from Definition 2.1, (iv), 
A new characterization of regularized BMO spaces on non-homogeneous spaces and its applications 13

(ii) and (iii) of Lemma 2.2 , that there exists a constant $C_{3} \in(1, \infty)$, depending on $\varrho$ and $\mu$, such that

$$
\begin{aligned}
\left|m_{f}\left(B_{x_{i}}^{*}\right)-m_{f}\left(\widetilde{5 B_{x_{i}}^{*}}\right)\right| & \leq\left[1+\delta\left(B_{x_{i}}^{*}, \widetilde{5 B_{x_{i}}^{*}}\right)\right]\|f\|_{\mathrm{RBMO}_{0, s}(\mu)} \\
& \leq\left[1+\delta\left(B_{x_{i}}^{*}, 5 B_{x_{i}}^{*}\right)+\delta\left(5 B_{x_{i}}^{*}, \widetilde{5 B_{x_{i}}^{*}}\right)\right]\|f\|_{\mathrm{RBMO}_{0, s}(\mu)} \\
& \leq C_{3}\|f\|_{\mathrm{RBMO}_{0, s}(\mu)}
\end{aligned}
$$

which, along with (2.10), implies that if $x \in 5 B_{x_{i}}^{*}$ satisfying $\left|f(x)-m_{f}\left(\widetilde{B_{0}}\right)\right|>k L$ with $k \geq 3$ and $L \geq 2 C_{3}\|f\|_{\mathrm{RBMO}_{0, s}(\mu)}$, then

$$
\begin{aligned}
& \left|f(x)-m_{f}\left(\widetilde{5 B_{x_{i}}^{*}}\right)\right| \\
& \geq\left|f(x)-m_{f}\left(\widetilde{B_{0}}\right)\right|-\left|m_{f}\left(\widetilde{B_{0}}\right)-m_{f}\left(B_{x_{i}}^{*}\right)\right|-\left|m_{f}\left(B_{x_{i}}^{*}\right)-m_{f}\left(\widetilde{5 B_{x_{i}}^{*}}\right)\right| \\
& >k L-\frac{5}{2} L-C_{3}\|f\|_{\mathrm{RBMO}_{0, s}(\mu)} \geq(k-3) L .
\end{aligned}
$$

Therefore,

$$
\begin{aligned}
& \left\{x \in B_{0}:\left|f(x)-m_{f}\left(\widetilde{B_{0}}\right)\right|>k L\right\} \\
& \subset \bigcup_{\left\{x \in B_{0},\left|f(x)-m_{f}\left(\widetilde{B_{0}}\right)\right|>k L\right\}}\left\{y \in B_{x}^{*}:\left|f(y)-m_{f}\left(\widetilde{B_{0}}\right)\right|>k L\right\} \\
& \subset \bigcup_{i}\left\{y \in 5 B_{x_{i}}^{*}:\left|f(y)-m_{f}\left(\widetilde{5 B_{x_{i}}^{*}}\right)\right|>(k-3) L\right\} .
\end{aligned}
$$

Using Definition 2.1, (iv), (ii) and (iii) of Lemma 2.2, we see that there exists a constant $C_{4} \in(1, \infty)$, depending on $\varrho$ and $\mu$, such that

$$
\begin{aligned}
& \left|m_{f}\left(\widetilde{\varrho B_{0}}\right)-m_{f}\left(\widetilde{B_{0}}\right)\right| \\
& \leq\left|m_{f}\left(\widetilde{\varrho B_{0}}\right)-m_{f}\left(B_{0}\right)\right|+\left|m_{f}\left(B_{0}\right)-m_{f}\left(\widetilde{B_{0}}\right)\right| \\
& \leq\left[2+\delta\left(B_{0}, \widetilde{\varrho B_{0}}\right)+\delta\left(B_{0}, \widetilde{B_{0}}\right)\right]\|f\|_{\mathrm{RBMO}_{0, s}(\mu)} \\
& \leq\left[2+\delta\left(B_{0}, \varrho B_{0}\right)+\delta\left(\varrho B_{0}, \widetilde{\varrho B_{0}}\right)+\delta\left(B_{0}, \widetilde{B_{0}}\right)\right]\|f\|_{\mathrm{RBMO}_{0, s}(\mu)} \\
& \leq C_{4}\|f\|_{\mathrm{RBMO}_{0, s}(\mu)} .
\end{aligned}
$$

Take $L$ such that $L \geq C_{4}\|f\|_{\mathrm{RBMO}_{0, s}(\mu)}$. Then by the facts that $\left\{B_{x_{i}}^{*}\right\}_{i}$ are $\left(6 \varrho^{2}, \beta_{6 \varrho^{2}}\right)$ doubling and disjoint, which are contained in $\varrho B_{0},(2.11)$ and $(2.4)$, we conclude that

$$
\begin{aligned}
\sum_{i} \mu\left(5 \varrho^{2} B_{x_{i}}^{*}\right) & \leq \sum_{i} \mu\left(6 \varrho^{2} B_{x_{i}}^{*}\right) \leq \beta_{6 \varrho^{2}} \sum_{i} \mu\left(B_{x_{i}}^{*}\right) \\
& \leq \frac{\beta_{6 \varrho^{2}}}{\gamma s} \sum_{i} \mu\left(\left\{y \in B_{x_{i}}^{*}:\left|f(y)-m_{f}\left(\widetilde{B_{0}}\right)\right|>2 L\right\}\right) \\
& \leq \frac{\beta_{6 \varrho^{2}}}{\gamma s} \mu\left(\left\{y \in \varrho B_{0}:\left|f(y)-m_{f}\left(\widetilde{B_{0}}\right)\right|>2 L\right\}\right)
\end{aligned}
$$




$$
\begin{aligned}
& \leq \frac{\beta_{6 \varrho^{2}}}{\gamma s} \mu\left(\left\{y \in \varrho B_{0}:\left|f(y)-m_{f}\left(\widetilde{\varrho B_{0}}\right)\right|+\left|m_{f}\left(\widetilde{\varrho B_{0}}\right)-m_{f}\left(\widetilde{B_{0}}\right)\right|>2 L\right\}\right) \\
& \leq \frac{\beta_{6 \varrho^{2}}}{\gamma s} \mu\left(\left\{y \in \varrho B_{0}:\left|f(y)-m_{f}\left(\widetilde{\varrho B_{0}}\right)\right|>L\right\}\right)<\frac{\beta_{6 \varrho^{2}}}{\gamma} \mu\left(\varrho^{2} B_{0}\right) .
\end{aligned}
$$

Therefore,

$$
\begin{aligned}
& \left\{x \in B_{0}:\left|f(x)-m_{f}\left(\widetilde{B_{0}}\right)\right|>k L\right\} \\
& \subset \bigcup_{i}\left\{y \in 5 B_{x_{i}}^{*}:\left|f(y)-m_{f}\left(\widetilde{5 B_{x_{i}}^{*}}\right)\right|>(k-3) L\right\}
\end{aligned}
$$

and

$$
\sum_{i} \mu\left(5 \varrho^{2} B_{x_{i}}^{*}\right) \leq \frac{\beta_{6 \varrho^{2}}}{\gamma} \mu\left(\varrho^{2} B_{0}\right)
$$

Denote $5 B_{x_{i}}^{*}$ simply by $B^{i}$. Let $n \in \mathbf{N}$. Iterating $n$ times with the balls $B^{i}$ in place of $B_{0}$, we see that

$$
\begin{aligned}
& \left\{x \in B_{0}:\left|f(x)-m_{f}\left(\widetilde{B_{0}}\right)\right|>3 n L\right\} \\
& \subset \bigcup_{i_{1}}\left\{y \in B^{i_{1}}:\left|f(y)-m_{f}\left(\widetilde{B^{i_{1}}}\right)\right|>3(n-1) L\right\} \\
& \subset \bigcup_{i_{1}, i_{2}}\left\{y \in B^{i_{1}, i_{2}}:\left|f(y)-m_{f}\left(\widetilde{B^{i_{1}, i_{2}}}\right)\right|>3(n-2) L\right\} \\
& \subset \cdots \subset \bigcup_{i_{1}, i_{2}, \cdots, i_{n}}\left\{y \in B^{i_{1}, i_{2}, \cdots, i_{n}}:\left|f(y)-m_{f}\left(\widetilde{B^{i_{1}, i_{2}, \cdots}, i_{n}}\right)\right|>0\right\}
\end{aligned}
$$

and hence

$$
\begin{aligned}
& \mu\left(\left\{x \in B_{0}:\left|f(x)-m_{f}\left(\widetilde{B_{0}}\right)\right|>3 n L\right\}\right) \leq \sum_{i_{1}, \cdots, i_{n-1}, i_{n}} \mu\left(B^{i_{1}, \cdots, i_{n-1}, i_{n}}\right) \\
& \leq \sum_{i_{1}, \cdots, i_{n-1}} \sum_{i_{n}} \mu\left(B^{i_{1}, \cdots, i_{n-1}, i_{n}}\right) \leq \frac{\beta_{6 \varrho^{2}}}{\gamma} \sum_{i_{1}, \cdots, i_{n-1}} \mu\left(\varrho^{2} B^{i_{1}, \cdots, i_{n-1}}\right) \\
& \leq \cdots \leq\left(\frac{\beta_{6 \varrho^{2}}}{\gamma}\right)^{n} \mu\left(\varrho^{2} B_{0}\right) .
\end{aligned}
$$

Take $L:=C_{5}\|f\|_{\mathrm{RBMO}_{0, s}(\mu)}$ with $C_{5}:=\max \left\{C_{1} / 2,2 C_{2}, 2 C_{3}, C_{4}\right\}$ and choose $n \in \mathbf{N}$ such that $t \in[3 n L, 3(n+1) L)$. We then know that

$$
\begin{aligned}
\mu\left(\left\{x \in B_{0}:\left|f(x)-m_{f}\left(\widetilde{B_{0}}\right)\right|>t\right\}\right) & \leq \mu\left(\left\{x \in B_{0}:\left|f(x)-m_{f}\left(\widetilde{B_{0}}\right)\right|>3 n L\right\}\right) \\
& \leq\left(\frac{\beta_{6 \varrho^{2}}}{\gamma}\right)^{n} \mu\left(\varrho^{2} B_{0}\right) \leq\left(\frac{\beta_{6 \varrho^{2}}}{\gamma}\right)^{\frac{t}{3 L}-1} \mu\left(\varrho^{2} B_{0}\right) \\
& \leq 2 e^{-\frac{(\ln 2) t}{3 C_{5}\|f\|_{\mathrm{RBMO}_{0, s}(\mu)}} \mu\left(\varrho^{2} B_{0}\right) .}
\end{aligned}
$$

This means that $(2.3)$ is true for any $t \in[3 L, \infty)$. 
A new characterization of regularized BMO spaces on non-homogeneous spaces and its applications 15

On the other hand, it is easy to show that for any $t \in(0,3 L)$,

$$
\begin{aligned}
& \mu\left(\left\{x \in B_{0}:\left|f(x)-m_{f}\left(\widetilde{B_{0}}\right)\right|>t\right\}\right) \leq \mu\left(\varrho^{2} B_{0}\right) \\
& \leq e^{\overline{3 C_{5}\|f\|_{\mathrm{RBMO}_{0, s}(\mu)}}} e^{-\frac{(\ln 2)}{3 C_{5}\|f\|_{\mathrm{RBMO}_{0, s}(\mu)}}} \mu\left(\varrho^{2} B_{0}\right) \leq 2 e^{-\frac{(\ln 2)}{3 C_{5}\|f\|_{\mathrm{RBMO}_{0, s}(\mu)}} \mu\left(\varrho^{2} B_{0}\right) .}
\end{aligned}
$$

Thus, (2.3) still holds for any $t \in(0,3 L)$, which completes the proof of Proposition 2.1 .

Based on Proposition 2.1, we now prove Theorem 2.1.

Proof of Theorem 2.1. We first show that if $f \in \operatorname{RBMO}(\mu)$, then $f \in \operatorname{RBMO}_{0, s}(\mu)$. Let $\varrho \in(1, \infty)$. For any ball $B \subset \mathcal{X}$, from the definition of $m_{0, s ; B}^{\varrho}\left(f-m_{f}(\widetilde{B})\right)$, we deduce that for any $t \in\left(0, m_{0, s ; B}^{\varrho}\left(f-m_{f}(\widetilde{B})\right)\right)$,

$$
\mu\left(\left\{y \in B:\left|f(y)-m_{f}(\widetilde{B})\right|>t\right\}\right) \geq s \mu(\varrho B)
$$

which implies that

$$
t \leq \frac{1}{s \mu(\varrho B)} \int_{B}\left|f(x)-m_{f}(\widetilde{B})\right| d \mu(x) .
$$

Letting $t \rightarrow m_{0, s ; B}^{\varrho}\left(f-m_{f}(\widetilde{B})\right)$, we then conclude that

$$
m_{0, s ; B}^{\varrho}\left(f-m_{f}(\widetilde{B})\right) \leq \frac{1}{s \mu(\varrho B)} \int_{B}\left|f(x)-m_{f}(\widetilde{B})\right| d \mu(x) .
$$

Choose $\rho=\varrho$ in Lemma 2.6. Then the above estimate, along with the fact that for any ball $B, c \in \mathbf{C}$ and $\mu$-measurable function $h, m_{h}(B)-c=m_{h-c}(B)$ and Lemmas 2.5 and 2.6, implies that

$$
\begin{aligned}
& m_{0, s ; B}^{\varrho}\left(f-m_{f}(\widetilde{B})\right) \\
& \leq \frac{1}{s \mu(\varrho B)} \int_{B}\left|f(x)-m_{\widetilde{B}}(f)\right| d \mu(x)+\frac{\mu(B)}{s \mu(\varrho B)}\left|m_{\widetilde{B}}(f)-m_{f}(\widetilde{B})\right| \\
& \leq \frac{1}{s \mu(\varrho B)} \int_{B}\left|f(x)-m_{\widetilde{B}}(f)\right| d \mu(x)+\left|m_{f-m_{f}(\widetilde{B})}(\widetilde{B})\right| \\
& \leq \frac{1}{s \mu(\varrho B)} \int_{B}\left|f(x)-m_{\widetilde{B}}(f)\right| d \mu(x)+m_{0, s ; \widetilde{B}}^{\varrho}\left(f-m_{f}(\widetilde{B})\right) \\
& \leq \frac{1}{s \mu(\varrho B)} \int_{B}\left|f(x)-m_{\widetilde{B}}(f)\right| d \mu(x)+\frac{1}{s \mu(\varrho \widetilde{B})} \int_{\widetilde{B}}\left|f(x)-m_{\widetilde{B}}(f)\right| d \mu(x) \\
& \leq s^{-1}\|f\|_{\operatorname{RBMO}(\mu)} .
\end{aligned}
$$

On the other hand, by the similar argument, we conclude that for all $\left(6 \varrho^{2}, \beta_{6 \varrho^{2}}\right)$ doubling balls $B \subset S \subset \mathcal{X}$, 


$$
\begin{aligned}
\left|m_{f}(B)-m_{f}(S)\right| \leq & \left|m_{f}(B)-m_{B}(f)\right|+\left|m_{B}(f)-m_{S}(f)\right|+\left|m_{S}(f)-m_{f}(S)\right| \\
\leq & \left|m_{f-m_{B}(f)}(B)\right|+\left|m_{B}(f)-m_{S}(f)\right|+\left|m_{f-m_{S}(f)}(S)\right| \\
\leq & m_{0, s ; B}^{\varrho}\left(f-m_{B}(f)\right)+[1+\delta(B, S)]\|f\|_{\operatorname{RBMO}(\mu)} \\
& +m_{0, s ; S}^{\varrho}\left(f-m_{S}(f)\right) \\
\leq & \frac{1}{s \mu(\varrho B)} \int_{B}\left|f(x)-m_{B}(f)\right| d \mu(x)+[1+\delta(B, S)]\|f\|_{\operatorname{RBMO}(\mu)} \\
& +\frac{1}{s \mu(\varrho S)} \int_{S}\left|f(x)-m_{S}(f)\right| d \mu(x) \\
\leq & \left(2 s^{-1}+1\right)\|f\|_{\operatorname{RBMO}(\mu) .}
\end{aligned}
$$

Therefore, for any $f \in \operatorname{RBMO}(\mu), f \in \mathrm{RBMO}_{0, s}(\mu)$ and $\|f\|_{\mathrm{RBMO}_{0, s}(\mu)} \lesssim\|f\|_{\mathrm{RBMO}(\mu)}$.

Now we prove that if $f \in \operatorname{RBMO}_{0, s}(\mu)$, then $f \in \operatorname{RBMO}(\mu)$ and

$$
\|f\|_{\mathrm{RBMO}(\mu)} \lesssim\|f\|_{\mathrm{RBMO}_{0, s}(\mu)} .
$$

To prove (2.12), we consider the following two cases.

Case I. $\|f\|_{\mathrm{RBMO}_{0, s}(\mu)}=0$. Just as in the proof of Proposition 2.1, we know that there exists a constant $M$ such that $f(x)=M$ for $\mu$-almost every $x \in \mathcal{X}$, which implies that $\|f\|_{\operatorname{RBMO}(\mu)}=0$.

Case II. $\|f\|_{\mathrm{RBMO}_{0, s}(\mu)}>0$. We now show (2.12). Indeed, by Definition 2.1 and Lemma 2.6, to prove (2.12), it suffices to show that

$$
\sup _{B \ni x} \frac{1}{\mu\left(\varrho^{2} B\right)} \int_{B}\left|f(x)-m_{\widetilde{B}}(f)\right| d \mu(x) \lesssim\|f\|_{\mathrm{RBMO}_{0, s}(\mu)},
$$

and that

$$
\sup _{\substack{x \in B \subset S \\ B, S\left(6 \varrho^{2}, \beta_{6 \varrho^{2}}\right)-\text { doubling }}}\left|m_{B}(f)-m_{S}(f)\right| \lesssim[1+\delta(B, S)]\|f\|_{\mathrm{RBMO}_{0, s}(\mu)} .
$$

With the aid of Proposition 2.1, we easily see that for all balls $B \subset \mathcal{X}$,

$$
\begin{aligned}
& \frac{1}{\mu\left(\varrho^{2} B\right)} \int_{B}\left|f(x)-m_{\widetilde{B}}(f)\right| d \mu(x) \\
\leq & \frac{1}{\mu\left(\varrho^{2} B\right)} \int_{B}\left|f(x)-m_{f}(\widetilde{B})\right| d \mu(x)+\left|m_{f}(\widetilde{B})-m_{\widetilde{B}}(f)\right| \\
\leq & \frac{1}{\mu\left(\varrho^{2} B\right)} \int_{B}\left|f(x)-m_{f}(\widetilde{B})\right| d \mu(x)+\frac{1}{\mu(\widetilde{B})} \int_{\widetilde{B}}\left|f(x)-m_{f}(\widetilde{B})\right| d \mu(x) \\
= & \frac{1}{\mu\left(\varrho^{2} B\right)} \int_{0}^{\infty} \mu\left(\left\{x \in B:\left|f(x)-m_{f}(\widetilde{B})\right|>t\right\}\right) d t \\
& +\frac{1}{\mu(\widetilde{B})} \int_{0}^{\infty} \mu\left(\left\{x \in \widetilde{B}:\left|f(x)-m_{f}(\widetilde{B})\right|>t\right\}\right) d t \\
\lesssim & \frac{1}{\mu\left(\varrho^{2} B\right)} \int_{0}^{\infty} \exp \left(-\frac{c_{\varrho} t}{\|f\|_{\mathrm{RBMO}_{0, s}(\mu)}}\right) \mu\left(\varrho^{2} B\right) d t \\
& +\frac{1}{\mu(\widetilde{B})} \int_{0}^{\infty} \exp \left(-\frac{c_{\varrho} t}{\|f\|_{\mathrm{RBMO}_{0, s}(\mu)}}\right) \mu(\widetilde{B}) d t \lesssim\|f\|_{\mathrm{RBMO}_{0, s}(\mu)},
\end{aligned}
$$


A new characterization of regularized BMO spaces on non-homogeneous spaces and its applications 17

which implies (2.13).

On the other hand, applying Proposition 2.1 again, we conclude that for all $\left(6 \varrho^{2}, \beta_{6 \varrho^{2}}\right)$-doubling balls $B \subset S \subset \mathcal{X}$,

$$
\begin{aligned}
\left|m_{B}(f)-m_{S}(f)\right| \leq & \left|m_{B}(f)-m_{f}(B)\right|+\left|m_{f}(B)-m_{f}(S)\right|+\left|m_{f}(S)-m_{S}(f)\right| \\
\leq & \frac{1}{\mu(B)} \int_{B}\left|f(x)-m_{f}(B)\right| d \mu(x)+[1+\delta(B, S)]\|f\|_{\mathrm{RBMO}_{0, s}(\mu)} \\
& +\frac{1}{\mu(S)} \int_{S}\left|f(x)-m_{f}(S)\right| d \mu(x) \\
= & \frac{1}{\mu(B)} \int_{0}^{\infty} \mu\left(\left\{x \in B:\left|f(x)-m_{f}(B)\right|>t\right\}\right) d t \\
& +[1+\delta(B, S)]\|f\|_{\mathrm{RBMO}_{0, s}(\mu)} \\
& +\frac{1}{\mu(S)} \int_{0}^{\infty} \mu\left(\left\{x \in S:\left|f(x)-m_{f}(S)\right|>t\right\}\right) d t \\
\lesssim & \frac{1}{\mu(B)} \int_{0}^{\infty} \exp \left(-\frac{c_{\varrho} t}{\|f\|_{\mathrm{RBMO}_{0, s}(\mu)}}\right) \mu\left(\varrho^{2} B\right) d t \\
& +[1+\delta(B, S)]\|f\|_{\mathrm{RBMO}_{0, s}(\mu)} \\
& +\frac{1}{\mu(S)} \int_{0}^{\infty} \exp \left(-\frac{c_{\varrho} t}{\|f\|_{\mathrm{RBMO}_{0, s}(\mu)}}\right) \mu\left(\varrho^{2} S\right) d t \\
\lesssim & {[1+\delta(B, S)]\|f\|_{\mathrm{RBMO}_{0, s}(\mu)}, }
\end{aligned}
$$

which implies (2.14). This finishes the proof of Theorem 2.1.

We end this section with the proof of Corollary 2.1.

Proof of Corollary 2.1. From the fact that $\varphi$ is strictly increasing, it follows that for any $t \in(0, \infty)$, any ball $B \subset \mathcal{X}$ and $y \in B$ such that $\left|f(y)-m_{f}(\widetilde{B})\right|>t$,

$$
\varphi\left(\left|f(y)-m_{f}(\widetilde{B})\right|\right)>\varphi(t) .
$$

This implies that

$$
\left\{y \in B:\left|f(y)-m_{f}(\widetilde{B})\right|>t\right\} \subset\left\{y \in B: \varphi\left(\left|f(y)-m_{f}(\widetilde{B})\right|\right)>\varphi(t)\right\} .
$$

Furthermore,

$$
\begin{aligned}
& \mu\left(\left\{y \in B:\left|f(y)-m_{f}(\widetilde{B})\right|>t\right\}\right) \\
& \leq \mu\left(\left\{y \in B: \varphi\left(\left|f(y)-m_{f}(\widetilde{B})\right|\right)>\varphi(t)\right\}\right) .
\end{aligned}
$$

On the other hand, by the definition of $m_{0, s ; B}^{\varrho}\left(f-m_{f}(\widetilde{B})\right)$, we see that for all $t \in$ $\left(0, m_{0, s ; B}^{\varrho}\left(f-m_{f}(\widetilde{B})\right)\right)$,

$$
\mu\left(\left\{y \in B:\left|f(y)-m_{f}(\widetilde{B})\right|>t\right\}\right) \geq s \mu(\varrho B)
$$

which, along with (2.15), yields

$$
\mu\left(\left\{y \in B: \varphi\left(\left|f(y)-m_{f}(\widetilde{B})\right|\right)>\varphi(t)\right\}\right) \geq s \mu(\varrho B) .
$$


Therefore,

$$
\varphi(t) \leq \frac{1}{s \mu(\varrho B)} \int_{B} \varphi\left(\left|f(y)-m_{f}(\widetilde{B})\right|\right) d \mu(y) .
$$

By the choice of the function $\varphi$, we easily know that the inverse function of $\varphi$ exists, which is denoted by $\varphi^{-1}$. From (2.16) and the facts that $\varphi$ is nonnegative continuous and

$$
\lim _{t \rightarrow \infty} \varphi(t)=\infty
$$

we infer that for all $t \in\left(0, m_{0, s ; B}^{\varrho}\left(f-m_{f}(\widetilde{B})\right)\right)$,

$$
t \leq \varphi^{-1}\left(\frac{1}{s \mu(\varrho B)} \int_{B} \varphi\left(\left|f(y)-m_{f}(\widetilde{B})\right|\right) d \mu(y)\right) .
$$

By letting $t \rightarrow m_{0, s ; B}^{\varrho}\left(f-m_{f}(\widetilde{B})\right)$, we conclude that

$$
m_{0, s ; B}^{\varrho}\left(f-m_{f}(\widetilde{B})\right) \leq \varphi^{-1}\left(\frac{1}{s \mu(\varrho B)} \int_{B} \varphi\left(\left|f(y)-m_{f}(\widetilde{B})\right|\right) d \mu(y)\right) .
$$

From this fact and Theorem 2.1, we deduce that if $f$ satisfies the assumptions of Corollary 2.1, then $f \in \mathrm{RBMO}_{0, s}(\mu)$. Hence, $f \in \operatorname{RBMO}(\mu)$, which completes the proof of Corollary 2.1.

\section{Some applications}

We begin this section with the notion of the Calderón-Zygmund operator on the non-homogeneous metric measure spaces.

Let $\Delta:=\{(x, x): x \in \mathcal{X}\}$ and $K$ be a $\mu$-locally integrable function mapping $(\mathcal{X} \times \mathcal{X}) \backslash \Delta$ to $\mathbf{C}$, which satisfies the size condition that there exists a positive constant $C$ such that for all $x, y \in \mathcal{X}$ with $x \neq y$,

$$
|K(x, y)| \leq C \frac{1}{\lambda(x, d(x, y))}
$$

and the regularity condition that there exist some positive constants $\tau$ and $C$ such that for all $x, x^{\prime}, y \in \mathcal{X}$ with $d(x, y) \geq 2 d\left(x, x^{\prime}\right)$,

$$
\left|K(x, y)-K\left(x^{\prime}, y\right)\right|+\left|K(y, x)-K\left(y, x^{\prime}\right)\right| \leq C \frac{\left[d\left(x, x^{\prime}\right)\right]^{\tau}}{[d(x, y)]^{\tau} \lambda(x, d(x, y))} .
$$

The Calderón-Zygmund operator associated to the above kernel $K$ and the measure $\mu$ is formally defined by

$$
T f(x):=\int_{\mathcal{X}} K(x, y) f(y) d \mu(y) .
$$

This integral may not be convergent for many functions. Thus, we consider the truncated operators $T_{\epsilon}$ for $\epsilon \in(0, \infty)$ defined by setting, for any suitable function $f$ and $x \in \mathcal{X}$,

$$
T_{\epsilon} f(x):=\int_{d(x, y)>\epsilon} K(x, y) f(y) d \mu(y) .
$$

Throughout this paper, we say that $T$ is bounded on $L^{p}(\mu)$ if the operators $T_{\epsilon}$ are bounded on $L^{p}(\mu)$ uniformly on $\epsilon \in(0, \infty)$, and $T$ satisfies some type of estimate if $T_{\epsilon}$ satisfies the same type of estimate uniformly on $\epsilon \in(0, \infty)$. 
A new example of operators with kernel satisfying (3.1) and (3.2) is the so-called Bergman-type operator appearing in [25]; see also [8] for an explanation. Notice that (3.1) and (3.2) are more general than the conditions satisfied by the classical Calderón-Zygmund operators.

The purpose of this section is to show that the $L^{p}(\mu)$-boundedness with $p \in(1, \infty)$ of the Calderón-Zygmund operator is equivalent to several corresponding endpoint estimates, based on the results obtained in Section 2. To this end, we first recall the definition of the atomic Hardy space $H^{1}(\mu)$ as follows.

Definition 3.1. Let $\rho \in(1, \infty)$ and $p \in(1, \infty]$. A function $b \in L_{\text {loc }}^{1}(\mu)$ is called

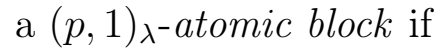

(i) there exists some ball $B \subset \mathcal{X}$ such that $\operatorname{supp}(b) \subset B$;

(ii) $\int_{\mathcal{X}} b(x) d \mu(x)=0$;

(iii) for $j \in\{1,2\}$, there exist a function $a_{j}$ supported on ball $B_{j} \subset B$ and a number $\kappa_{j} \in \mathbf{R}$ such that $b=\kappa_{1} a_{1}+\kappa_{2} a_{2}$ and

$$
\left\|a_{j}\right\|_{L^{p}(\mu)} \leq\left[\mu\left(\rho B_{j}\right)\right]^{1 / p-1}\left[1+\delta\left(B_{j}, B\right)\right]^{-1} .
$$

Then define $|b|_{H_{\mathrm{ath}}^{1, p}(\mu)}:=\left|\kappa_{1}\right|+\left|\kappa_{2}\right|$. A function $f \in L^{1}(\mu)$ is said to belong to the space $H_{\text {atb }}^{1, p}(\mu)$ if there exist $(p, 1)_{\lambda}$-atomic blocks $\left\{b_{i}\right\}_{i \in \mathbf{N}}$ such that $f=\sum_{i=1}^{\infty} b_{i}$ with $\sum_{i=1}^{\infty}\left|b_{i}\right|_{H_{\mathrm{atb}}^{1, p}(\mu)}<\infty$. The $H_{\mathrm{atb}}^{1, p}(\mu)$ norm of $f$ is defined by

$$
\|f\|_{H_{\mathrm{atb}}^{1, p}(\mu)}:=\inf \left\{\sum_{i=1}^{\infty}\left|b_{i}\right|_{H_{\mathrm{atb}}^{1, p}(\mu)}\right\},
$$

where the infimum is taken over all the possible decompositions of $f$ into $(p, 1)_{\lambda^{-}}$ atomic blocks.

It was proved in $[9$, Proposition 3.1(ii) $]$ that for each $p \in(1, \infty]$, the space $H_{\text {atb }}^{1, p}(\mu)$ is independent of the choice of $\rho \in(1, \infty)$ and, for all $p \in(1, \infty)$, the spaces $H_{\text {atb }}^{1, p}(\mu)$ and $H_{\mathrm{atb}}^{1, \infty}(\mu)$ coincide with equivalent norms. Thus, in this paper, we write $H_{\mathrm{atb}}^{1, p}(\mu)$ simply by $H^{1}(\mu)$.

The following theorem is the main result of this section.

Theorem 3.1. Let $\rho \in(1, \infty), K$ be a $\mu$-locally integrable function mapping $(\mathcal{X} \times \mathcal{X}) \backslash \Delta$ to $\mathbf{C}$ which satisfies (3.1) and (3.2), and T a Calderón-Zygmund operator as in (3.3). Then the following seven statements are equivalent:

(i) $T$ is bounded from $H^{1}(\mu)$ into $L^{1}(\mu)$;

(ii) $T$ is bounded from $H^{1}(\mu)$ into weak $L^{1}(\mu)$;

(iii) for some $\nu \in(0, \infty)$, there exists a positive constant $C$ such that for all $\epsilon, t \in(0, \infty)$, balls $B$ and bounded functions $f$ with $\operatorname{supp}(f) \subset B$,

$$
\mu\left(\left\{x \in B:\left|T_{\epsilon}(f)(x)\right|>t\right\}\right) \leq C t^{-\nu} \mu(\rho B)\|f\|_{L^{\infty}(\mu)}^{\nu} ;
$$

(iv) for some $\sigma \in(0,1)$, there exists a positive constant $C$ such that for all $\epsilon \in$ $(0, \infty)$, balls $B$ and bounded functions $f$ with $\operatorname{supp}(f) \subset B$,

$$
\frac{1}{\mu(\rho B)} \int_{B}\left|T_{\epsilon}(f)(x)\right|^{\sigma} d \mu(x) \leq C\|f\|_{L^{\infty}(\mu)}^{\sigma} ;
$$

(v) $T$ is bounded from $L^{\infty}(\mu)$ into $\operatorname{RBMO}(\mu)$;

(vi) $T$ is bounded on $L^{p}(\mu)$ for some $p \in(1, \infty)$;

(vii) $T$ is bounded on $L^{p}(\mu)$ for all $p \in(1, \infty)$. 
When $(\mathcal{X}, d, \mu):=\left(\mathbf{R}^{d},|\cdot|, \mu\right)$ with $\mu$ satisfying the polynomial growth condition (1.2), the corresponding result was obtained in [5]. Furthermore, it was already proved in [15] that for the Calderón-Zygmund operator $T$ on non-homogeneous spaces, its boundedness on $L^{p}(\mu)$ with some $p \in(1, \infty)$ is equivalent to its boundedness from $H^{1}(\mu)$ into weak $L^{1}(\mu)$. To be precise, (i) $\Leftrightarrow$ (ii) $\Leftrightarrow$ (vi) and (ii) $\Rightarrow$ (iii) $\Rightarrow$ (iv) have been proved in [15]. In this article, applying the new characterization of the space $\operatorname{RBMO}(\mu)$ in Section 2 (to be precise, Corollary 2.1), we prove the implication (iv) $\Rightarrow(\mathrm{v})$.

From Theorem 3.1, we further deduce the following result.

Corollary 3.1. Let $K$ be a $\mu$-locally integrable function mapping $(\mathcal{X} \times \mathcal{X}) \backslash \Delta$ to $\mathbf{C}$ which satisfies (3.1) and (3.2), and $T$ a Calderón-Zygmund operator as in (3.3). Let $\Phi$ be a Young function such that for all $t_{1}, t_{2} \in[0, \infty), \Phi\left(t_{1} t_{2}\right) \leq C_{\Phi} \Phi\left(t_{1}\right) \Phi\left(t_{2}\right)$, where $C_{\Phi}$ is a positive constant independent of $t_{1}$ and $t_{2}$, and that for some $\sigma \in(0,1)$,

$$
\int_{0}^{\infty} \Phi\left(\frac{1}{t}\right) t^{\sigma-1} d t<\infty
$$

If there exists a positive constant $C$ such that for all $\epsilon, t \in(0, \infty)$ and bounded functions $f$ with bounded support,

$$
\mu\left(\left\{x \in \mathcal{X}:\left|T_{\epsilon}(f)(x)\right|>t\right\}\right) \leq C \int_{\mathcal{X}} \Phi\left(\frac{|f(x)|}{t}\right) d \mu(x),
$$

then $T$ is bounded on $L^{p}(\mu)$ for all $p \in(1, \infty)$.

We remark that if we let $\Phi(t):=t \log ^{\gamma}(2+t)$ with $\gamma \in[1, \infty)$ for all $t \in[0, \infty)$, then by Corollary 3.1, we easily see that if $T$ is of weak type $\left(L \log ^{\gamma} L(\mu), L^{1}(\mu)\right)$, namely, there exists a positive constant $C$ such that for all $t \in(0, \infty)$ and bounded functions $f$ with bounded support,

$$
\mu\left(\left\{x \in \mathcal{X}:\left|T_{\epsilon}(f)(x)\right|>t\right\}\right) \leq C \int_{\mathcal{X}} \frac{|f(x)|}{t} \log ^{\gamma}\left(\mathrm{e}+\frac{|f(x)|}{t}\right) d \mu(x),
$$

then $T$ is also bounded on $L^{p}(\mu)$ for all $p \in(1, \infty)$.

To prove Theorem 3.1, we need the following technical lemma.

Lemma 3.1. Let $K$ be a $\mu$-locally integrable function mapping $(\mathcal{X} \times \mathcal{X}) \backslash \Delta$ to $\mathbf{C}$ which satisfies (3.1) and (3.2), and $T$ a Calderón-Zygmund operator as in (3.3). Let $\sigma \in(0,1)$. If Theorem 3.1(iv) with $\rho=3 / 2$ is true, then for all $\epsilon \in$ $(0, \infty)$ and bounded functions $f$ with bounded support, $\left|T_{\epsilon}(f)\right|^{\sigma} \in \operatorname{RBMO}(\mu)$ and $\left\|\left|T_{\epsilon}(f)\right|^{\sigma}\right\|_{\operatorname{RBMO}(\mu)} \leq C\|f\|_{L^{\infty}(\mu)}^{\sigma}$, where $C$ is a positive constant independent of $f$ and $\epsilon$.

Proof. By the homogeneity of $\|\cdot\|_{\operatorname{RBMO}(\mu)}$, we may assume that $\|f\|_{L^{\infty}(\mu)}=1$. For any ball $B \subset \mathcal{X}$, set

$$
h_{B, \sigma}:=m_{B}\left(\left|T_{\epsilon}\left(f \chi_{\mathcal{X} \backslash \frac{4}{3} B}\right)\right|^{\sigma}\right),
$$

where for any $\mu$-locally integrable function $g$ and any ball $B \subset \mathcal{X}, m_{B}(g)$ denotes the mean of $g$ over $B$, namely, $m_{B}(g):=\frac{1}{\mu(B)} \int_{B} g(x) d \mu(x)$. It follows, from Definition 1.3, that the proof of Lemma 3.1 can be reduced to proving that

$$
\left.\frac{1}{\mu(2 B)} \int_{B}|| T_{\epsilon}(f)(x)\right|^{\sigma}-h_{B, \sigma} \mid d \mu(x) \lesssim 1,
$$


A new characterization of regularized BMO spaces on non-homogeneous spaces and its applications 21 and that for all balls $B$ and $S$ with $B \subset S$,

$$
\left|h_{B, \sigma}-h_{S, \sigma}\right| \lesssim 1+\delta(B, S) \text {. }
$$

We first show (3.5). For any fixed ball $B$ and bounded function $f$ with bounded support and $\|f\|_{L^{\infty}(\mu)}=1$, decompose $f$ as $f=f \chi_{\frac{4}{3} B}+f \chi_{\mathcal{X} \backslash \frac{4}{3} B}=: f_{1}+f_{2}$. Write

$$
\begin{aligned}
& \left.\frac{1}{\mu(2 B)} \int_{B}|| T_{\epsilon}(f)(x)\right|^{\sigma}-h_{B, \sigma} \mid d \mu(x) \\
& \leq\left.\frac{1}{\mu(2 B)} \int_{B}|| T_{\epsilon}(f)(x)\right|^{\sigma}-\left|T_{\epsilon}\left(f_{2}\right)(x)\right|^{\sigma}\left|d \mu(x)+\frac{1}{\mu(2 B)} \int_{B}\right|\left|T_{\epsilon}\left(f_{2}\right)(x)\right|^{\sigma}-h_{B, \sigma} \mid d \mu(x) \\
& \leq \frac{1}{\mu(2 B)} \int_{B}\left|T_{\epsilon}\left(f_{1}\right)(x)\right|^{\sigma} d \mu(x)+\left.\frac{1}{\mu(2 B)} \int_{B}|| T_{\epsilon}\left(f_{2}\right)(x)\right|^{\sigma}-h_{B, \sigma} \mid d \mu(x) .
\end{aligned}
$$

By the hypothesis, we easily know that

$$
\frac{1}{\mu(2 B)} \int_{B}\left|T_{\epsilon}\left(f_{1}\right)(x)\right|^{\sigma} d \mu(x) \leq \frac{1}{\mu(2 B)} \int_{\frac{4}{3} B}\left|T_{\epsilon}\left(f_{1}\right)(x)\right|^{\sigma} d \mu(x) \lesssim 1 .
$$

On the other hand, from (3.1) and (3.2), it follows that for all $x, y \in B$,

$$
\left.|| T_{\epsilon}\left(f_{2}\right)(x)\right|^{\sigma}-\left|T_{\epsilon}\left(f_{2}\right)(y)\right|^{\sigma}|\leq| T_{\epsilon}\left(f_{2}\right)(x)-\left.T_{\epsilon}\left(f_{2}\right)(y)\right|^{\sigma} \lesssim\|f\|_{L^{\infty}(\mu)}^{\sigma} \lesssim 1,
$$

which implies that

$$
\begin{aligned}
& \left.\frac{1}{\mu(2 B)} \int_{B}|| T_{\epsilon}\left(f_{2}\right)(x)\right|^{\sigma}-h_{B, \sigma} \mid d \mu(x) \\
& \leq\left.\frac{1}{\mu(2 B)} \frac{1}{\mu(B)} \int_{B} \int_{B}|| T_{\epsilon}\left(f_{2}\right)(x)\right|^{\sigma}-\left|T_{\epsilon}\left(f_{2}\right)(y)\right|^{\sigma} \mid d \mu(y) d \mu(x) \lesssim 1 .
\end{aligned}
$$

Therefore, (3.5) holds.

We now turn to prove (3.6). For all balls $B$ and $S$ with $B \subset S$, we denote the smallest positive integer $k$ such that $\frac{4}{3} S \subset 2^{k} B$ by $N$. Write

$$
\begin{aligned}
\left|h_{B, \sigma}-h_{S, \sigma}\right| \leq & \left|m_{B}\left(\left|T_{\epsilon}\left(f \chi_{\mathcal{X} \backslash \frac{4}{3} B}\right)\right|^{\sigma}\right)-m_{B}\left(\left|T_{\epsilon}\left(f \chi_{\mathcal{X} \backslash 2^{N} B}\right)\right|^{\sigma}\right)\right| \\
& +\left|m_{B}\left(\left|T_{\epsilon}\left(f \chi_{\mathcal{X} \backslash 2^{N} B}\right)\right|^{\sigma}\right)-m_{S}\left(\left|T_{\epsilon}\left(f \chi_{\mathcal{X} \backslash 2^{N} B}\right)\right|^{\sigma}\right)\right| \\
& +\left|m_{S}\left(\left|T_{\epsilon}\left(f \chi_{\mathcal{X} \backslash \frac{4}{3} S}\right)\right|^{\sigma}\right)-m_{S}\left(\left|T_{\epsilon}\left(f \chi_{\mathcal{X} \backslash 2^{N} B}\right)\right|^{\sigma}\right)\right| \\
\leq & m_{B}\left(\left|T_{\epsilon}\left(f \chi_{2^{N} B \backslash \frac{4}{3} B}\right)\right|^{\sigma}\right)+\mid m_{B}\left(\left|T_{\epsilon}\left(f \chi_{\mathcal{X} \backslash 2^{N} B}\right)\right|^{\sigma}\right) \\
& -m_{S}\left(\left|T_{\epsilon}\left(f \chi_{\mathcal{X} \backslash 2^{N} B}\right)\right|^{\sigma}\right) \mid+m_{R}\left(\left|T_{\epsilon}\left(f \chi_{2^{N} B \backslash \frac{4}{3} S}\right)\right|^{\sigma}\right) \\
= & : \mathrm{E}_{1}+\mathrm{E}_{2}+\mathrm{E}_{3} .
\end{aligned}
$$

By (3.1), we first conclude that for all $x \in B$,

$$
\begin{aligned}
\left|T_{\epsilon}\left(f \chi_{2^{N} B \backslash \frac{4}{3} B}\right)(x)\right| \lesssim & \int_{2^{N} B \backslash 2 B} \frac{|f(y)|}{\lambda(x, d(x, y))} d \mu(y) \\
& +\int_{2 B \backslash \frac{4}{3} B} \frac{|f(y)|}{\lambda(x, d(x, y))} d \mu(y) \lesssim 1+\delta(B, S),
\end{aligned}
$$


which, along with the fact $\sigma \in(0,1)$, implies that

$$
\mathrm{E}_{1} \lesssim[1+\delta(B, S)]^{\sigma} \leq 1+\delta(B, S)
$$

Analogously, $\mathrm{E}_{3} \lesssim 1$. It follows, from (3.1) and (3.2), that for all $x \in B$ and $y \in S$, $\left.|| T_{\epsilon}\left(f \chi_{\mathcal{X} \backslash 2^{N} B}\right)(x)\right|^{\sigma}-\left|T_{\epsilon}\left(f \chi_{\mathcal{X} \backslash 2^{N} B}\right)(y)\right|^{\sigma}|\leq| T_{\epsilon}\left(f \chi_{\mathcal{X} \backslash 2^{N} B}\right)(x)-\left.T_{\epsilon}\left(f \chi_{\mathcal{X} \backslash 2^{N} B}\right)(y)\right|^{\sigma} \lesssim 1$.

This further shows that

$$
\mathrm{E}_{2} \leq\left.\frac{1}{\mu(B)} \frac{1}{\mu(S)} \int_{B} \int_{S}|| T_{\epsilon}\left(f \chi_{\mathcal{X} \backslash 2^{N} B}\right)(x)\right|^{\sigma}-\left|T_{\epsilon}\left(f \chi_{\mathcal{X} \backslash 2^{N} B}\right)(y)\right|^{\sigma} \mid d \mu(y) d \mu(x) \lesssim 1 .
$$

By the estimates for $E_{1}, E_{2}$ and $E_{3}$, we obtain (3.6), which completes the proof of Lemma 3.1.

Proof of Theorem 3.1. By [15, Theorem 1.1, Corollary 1.1 and Lemma 3.1], we know that (i) $\Leftrightarrow$ (ii) $\Leftrightarrow$ (vi) and (ii) $\Rightarrow$ (iii) $\Rightarrow$ (iv). Now we prove that (iv) $\Rightarrow(\mathrm{v}) \Rightarrow$ (i), which implies the statements (i)-(vi) are equivalent. We then finally show that (vi) $\Leftrightarrow$ (vii).

(iv) $\Rightarrow(\mathrm{v})$. For simplicity, assume that (iv) is true with $\rho=3 / 2$. To show (v), it suffices to prove that for all $\epsilon \in(0, \infty)$ and bounded functions $f$ with bounded support,

$$
\left\|T_{\epsilon}(f)\right\|_{\operatorname{RBMO}(\mu)} \lesssim\|f\|_{L^{\infty}(\mu)} .
$$

It follows, from Lemma 3.1, that $\left|T_{\epsilon}(f)\right|^{\sigma} \in \operatorname{RBMO}(\mu)$, where $\sigma \in(0,1)$. By the John-Nirenberg inequality in [7], we know that $T_{\epsilon} f$ is $\mu$-locally integrable. For each fixed ball $B$, set $h_{B}:=m_{B}\left(T_{\epsilon}\left(f \chi_{\mathcal{X} \backslash \frac{\varrho+1}{2} B}\right)\right)$. By some arguments similar to those used in the proof of Lemma 3.1, we conclude that for all balls $B$,

$$
\frac{1}{\mu(\varrho B)} \int_{B}\left|T_{\epsilon}(f)(x)-h_{B}\right|^{\sigma} d \mu(x) \lesssim\|f\|_{L^{\infty}(\mu)}^{\sigma},
$$

and that for all balls $B$ and $S$ with $B \subset S$,

$$
\left|h_{B}-h_{S}\right| \lesssim[1+\delta(B, S)]\|f\|_{L^{\infty}(\mu)} .
$$

Notice that for any ball $B, c \in \mathbf{C}$ and $\mu$-measurable function $h, m_{h}(B)-c=m_{h-c}(B)$. From this fact, (3.7), (3.8) and (2.2), it follows that for all balls $B$,

$$
\begin{aligned}
& \int_{B}\left|T_{\epsilon}(f)(y)-m_{T_{\epsilon}(f)}(\widetilde{B})\right|^{\sigma} d \mu(y) \\
& \leq \int_{B}\left|T_{\epsilon}(f)(y)-h_{B}\right|^{\sigma} d \mu(y)+\left|h_{B}-h_{\widetilde{B}}\right|^{\sigma} \mu(B)+\left|h_{\widetilde{B}}-m_{T_{\epsilon}(f)}(\widetilde{B})\right|^{\sigma} \mu(B) \\
& \lesssim \mu(\varrho B)\|f\|_{L^{\infty}(\mu)}^{\sigma}+[1+\delta(B, \widetilde{B})]^{\sigma} \mu(B)\|f\|_{L^{\infty}(\mu)}^{\sigma}+\left|m_{\left[T_{\epsilon}(f)-h_{\widetilde{B}}\right]}(\widetilde{B})\right|^{\sigma} \mu(B) \\
& \lesssim \mu(\varrho B)\|f\|_{L^{\infty}(\mu)}^{\sigma}+\left(m_{0, s ; \widetilde{B}}^{\varrho}\left[T_{\epsilon}(f)-h_{\widetilde{B}}\right]\right)^{\sigma} \mu(B) \\
& \lesssim \mu(\varrho B)\|f\|_{L^{\infty}(\mu)}^{\sigma}+\frac{\mu(B)}{\mu(\varrho \widetilde{B})} \int_{\widetilde{B}}\left|T_{\epsilon}(f)(y)-h_{\widetilde{B}}\right|^{\sigma} d \mu(y) \lesssim \mu(\varrho B)\|f\|_{L^{\infty}(\mu)}^{\sigma},
\end{aligned}
$$


A new characterization of regularized BMO spaces on non-homogeneous spaces and its applications 23 and that for any two $\left(6 \varrho^{2}, \beta_{6 \varrho^{2}}\right)$-doubling balls $B \subset S$,

$$
\begin{aligned}
\left|m_{T_{\epsilon}(f)}(B)-m_{T_{\epsilon}(f)}(S)\right| \leq & \left|m_{T_{\epsilon}(f)}(B)-h_{B}\right|+\left|h_{B}-h_{S}\right|+\left|m_{T_{\epsilon}(f)}(S)-h_{S}\right| \\
\lesssim & \left|m_{\left[T_{\epsilon}(f)-h_{B}\right]}(B)\right|+[1+\delta(B, S)]\|f\|_{L^{\infty}(\mu)}+\left|m_{\left[T_{\epsilon}(f)-h_{S}\right]}(S)\right| \\
\lesssim & m_{0, s ; B}^{\varrho}\left(T_{\epsilon}(f)-h_{B}\right)+[1+\delta(B, S)]\|f\|_{L^{\infty}(\mu)} \\
& +m_{0, s ; S}^{\varrho}\left(T_{\epsilon}(f)-h_{S}\right) \\
\lesssim & {\left[\frac{1}{\mu(\varrho B)} \int_{B}\left|T_{\epsilon}(f)(y)-h_{B}\right|^{\sigma} d \mu(y)\right]^{1 / \sigma} } \\
& +[1+\delta(B, S)]\|f\|_{L^{\infty}(\mu)} \\
& +\left[\frac{1}{\mu(\varrho S)} \int_{S}\left|T_{\epsilon}(f)(y)-h_{S}\right|^{\sigma} d \mu(y)\right]^{1 / \sigma} \\
\lesssim & {[1+\delta(B, S)]\|f\|_{L^{\infty}(\mu),} }
\end{aligned}
$$

where $\widetilde{B}$ is the smallest $\left(6 \varrho^{2}, \beta_{6 \varrho^{2}}\right)$-doubling ball of the form $\left(6 \varrho^{2}\right)^{j} B$ with $j \in \mathbf{Z}_{+}$ as in Section 2. By Corollary 2.1 with $\varphi(t):=t^{\sigma}$ for all $t \in[0, \infty)$, we see that $\left\|T_{\epsilon}(f)\right\|_{\mathrm{RBMO}(\mu)} \lesssim\|f\|_{L^{\infty}(\mu)}$. Thus, (v) holds.

$(\mathrm{v}) \Rightarrow(\mathrm{i})$. We first claim that for all $\epsilon \in(0, \infty)$, balls $B$ and bounded functions $f$ with support contained in $B$,

$$
\int_{B}\left|T_{\epsilon}(f)(x)\right| d \mu(x) \lesssim \mu(2 B)\|f\|_{L^{\infty}(\mu)} .
$$

We consider the following two cases for $r_{B}$.

Case I. $r_{B} \leq \operatorname{diam}(\operatorname{supp} \mu) / 40$. In this case, choose $\rho=2$ and $\varrho=1$ in Lemma 2.6. From the hypothesis and Lemma 2.6, it follows that for all $\epsilon \in(0, \infty)$,

$$
\int_{B}\left|T_{\epsilon}(f)(x)-m_{\widetilde{B^{6}}}\left(T_{\epsilon}(f)\right)\right| d \mu(x) \lesssim \mu(2 B)\|f\|_{L^{\infty}(\mu)},
$$

where for any ball $B \subset \mathcal{X}, \widetilde{B^{6}}$ denotes the smallest $\left(6, \beta_{6}\right)$-doubling ball of the form $6^{j} B$ with $j \in \mathbf{Z}_{+}$. Hence, in this case, the proof of (3.9) is reduced to showing

$$
\left|m_{\widetilde{B^{6}}}\left(T_{\epsilon}(f)\right)\right| \lesssim \mu(2 B)\|f\|_{L^{\infty}(\mu)} .
$$

We use the same notation as in the proof of [15, Lemma 3.1]. Let $S$ be the smallest ball of the form $6^{j} B$ such that $\mu\left(6^{j} B \backslash 2 B\right)>0$ with $j \in \mathbf{N}$. Thus, $\mu\left(6^{-1} S \backslash 2 B\right)=0$ and $\mu(S \backslash 2 B)>0$. This leads to $\mu\left(S \backslash\left(6^{-1} S \cup 2 B\right)\right)>0$ and $\widetilde{B^{6}} \subset \widetilde{S^{6}}$. By this and [7, Lemma 3.3], we choose $\left.x_{0} \in S \backslash\left(6^{-1} S \cup 2 B\right)\right)$ such that the ball center at $x_{0}$ with the radius $6^{-k} r_{S}$ for some integer $k \geq 2$ is $\left(6, \beta_{6}\right)$-doubling. Let $B_{0}$ be the largest ball of this form. Then it is easy to show that $B_{0} \subset 2 S$ and $d\left(B_{0}, B\right) \geq r_{B} / 2$. It was proved in the proof of [15, Lemma 3.1] that $\delta(B, 2 S) \lesssim 1$ and $\delta\left(B_{0}, 2 S\right) \lesssim 1$, which imply that $\delta\left(B, \widetilde{(2 S)^{6}}\right) \lesssim 1$ and $\delta\left(B_{0}, \widetilde{(2 S)^{6}}\right) \lesssim 1$. Therefore, via Lemma 2.6, we conclude that

$$
\begin{aligned}
& \left|m_{B_{0}}\left(T_{\epsilon}(f)\right)-m_{\widetilde{B^{6}}}\left(T_{\epsilon}(f)\right)\right| \\
& \leq\left|m_{B_{0}}\left(T_{\epsilon}(f)\right)-m_{\widetilde{(2 S)^{6}}}\left(T_{\epsilon}(f)\right)\right|+\left|m_{\widetilde{(2 S)^{6}}}\left(T_{\epsilon}(f)\right)-m_{\widetilde{B^{6}}}\left(T_{\epsilon}(f)\right)\right| \\
& \leq\left[2+\delta\left(B_{0}, \widetilde{(2 S)^{6}}\right)+\delta\left(\widetilde{B^{6}}, \widetilde{(2 S)^{6}}\right)\right]\left\|T_{\epsilon}(f)\right\|_{\mathrm{RBMO}(\mu)} \lesssim\|f\|_{L^{\infty}(\mu)},
\end{aligned}
$$


which implies that to prove (3.10), it suffices to show

$$
\left|m_{B_{0}}\left(T_{\epsilon}(f)\right)\right| \lesssim\|f\|_{L^{\infty}(\mu)} .
$$

Notice that for all $y \in B_{0}$ and $z \in B, d(y, z) \geq r_{B} / 2$ and hence $d\left(x_{B}, y\right) \leq d\left(x_{B}, z\right)+$ $d(z, y) \lesssim d(z, y)$. From this, (3.1) and (1.4), it follows that for all $y \in B_{0}$,

$$
\begin{aligned}
\left|T_{\epsilon}(f)(y)\right| & \lesssim \int_{B} \frac{|f(z)|}{\lambda(y, d(y, z))} d \mu(z) \lesssim \int_{B} \frac{|f(z)|}{\lambda\left(x_{B}, d(y, z)\right)} d \mu(z) \\
& \lesssim \frac{\mu(B)}{\lambda\left(x_{B}, r_{B}\right)}\|f\|_{L^{\infty}(\mu)} \lesssim\|f\|_{L^{\infty}(\mu)},
\end{aligned}
$$

which implies (3.11). Therefore, (3.9) holds in this case.

Case II. $r_{B}>\operatorname{diam}(\operatorname{supp} \mu) / 40$. In this case, without loss of generality, we may assume $r_{B} \leq 8 \operatorname{diam}(\operatorname{supp} \mu)$. Then $B \cap \operatorname{supp}(\mu)$ is covered by balls $\left\{B_{j}\right\}_{j=1}^{M}$ with radius $r_{B} / 400$, where $M \in \mathbf{N}$. For $j \in\{1, \cdots, M\}$, define

$$
a_{j}:=\frac{\chi_{B_{j}}}{\sum_{k=1}^{M} \chi_{B_{k}}} f
$$

Since (3.9) holds if we replace $B$ by $2 B_{j}$, which supports the function $a_{j}$, we then see that

$$
\begin{aligned}
\int_{B}\left|T_{\epsilon}(f)(x)\right| d \mu(x) & \leq \sum_{j=1}^{M} \int_{B \backslash 2 B_{j}}\left|T_{\epsilon}\left(a_{j}\right)(x)\right| d \mu(x)+\sum_{j=1}^{M} \int_{2 B_{j}}\left|T_{\epsilon}\left(a_{j}\right)(x)\right| d \mu(x) \\
& \lesssim \sum_{j=1}^{M}\left\|a_{j}\right\|_{L^{\infty}(\mu)} \mu(B)+\sum_{j=1}^{M}\left\|a_{j}\right\|_{L^{\infty}(\mu)} \mu\left(4 B_{j}\right) \lesssim\|f\|_{L^{\infty}(\mu)} \mu(2 B) .
\end{aligned}
$$

Thus, the claim (3.9) also holds in this case.

Now based on the claim (3.9), we prove (i). Take $\rho=4$ and $p=\infty$ in Definition 3.1. It suffices to show that for all $(\infty, 1)_{\lambda}$-atomic blocks $b$,

$$
\left\|T_{\epsilon}(b)\right\|_{L^{1}(\mu)} \lesssim|b|_{H_{\mathrm{atb}}^{1, \infty}(\mu)} .
$$

Let $b:=\sum_{j=1}^{2} \kappa_{j} a_{j}$ be a $(\infty, 1)_{\lambda}$-atomic block, where for any $j \in\{1,2\}, \operatorname{supp}\left(a_{j}\right) \subset$ $B_{j} \subset B$ for some $B_{j}$ and $B$ as in Definition 3.1. Write

$$
\begin{aligned}
\left\|T_{\epsilon}(b)\right\|_{L^{1}(\mu)}= & \int_{2 B}\left|T_{\epsilon}(b)(x)\right| d \mu(x)+\int_{\mathcal{X} \backslash(2 B)} \ldots \\
\leq & \sum_{j=1}^{2}\left|\kappa_{j}\right| \int_{2 B_{j}}\left|T_{\epsilon}\left(a_{j}\right)(x)\right| d \mu(x)+\sum_{j=1}^{2}\left|\kappa_{j}\right| \int_{2 B \backslash 2 B_{j}} \ldots \\
& +\int_{\mathcal{X} \backslash(2 B)}\left|T_{\epsilon}(b)(x)\right| d \mu(x)=: \sum_{i=1}^{3} \mathrm{~F}_{i} .
\end{aligned}
$$

It follows, from (3.9), that

$$
\mathrm{F}_{1} \lesssim \sum_{j=1}^{2}\left|\kappa_{j}\right| \mu\left(4 B_{j}\right)\|a\|_{L^{\infty}(\mu)} \lesssim \sum_{j=1}^{2}\left|\kappa_{j}\right| .
$$


A new characterization of regularized BMO spaces on non-homogeneous spaces and its applications 25

On the other hand, by (3.1) and (1.4), we conclude that

$$
\begin{aligned}
\mathrm{F}_{2} & \lesssim \sum_{j=1}^{2}\left|\kappa_{j}\right| \int_{(2 B) \backslash\left(2 B_{j}\right)} \int_{B_{j}} \frac{\left|a_{j}(y)\right|}{\lambda(x, d(x, y))} d \mu(y) d \mu(x) \\
& \lesssim \sum_{j=1}^{2}\left|\kappa_{j}\right| \int_{(2 B) \backslash\left(2 B_{j}\right)} \int_{B_{j}} \frac{\left|a_{j}(y)\right|}{\lambda\left(x_{B_{j}}, d\left(x, x_{B_{j}}\right)\right)} d \mu(y) d \mu(x) \\
& \lesssim \sum_{j=1}^{2}\left|\kappa_{j}\right| \delta\left(B_{j}, B\right)\left\|a_{j}\right\|_{L^{1}(\mu)} \lesssim \sum_{j=1}^{2}\left|\kappa_{j}\right| .
\end{aligned}
$$

It remain to estimate $\mathrm{F}_{3}$. We consider the following two cases.

Case (i). $\epsilon \in\left(0, r_{B}\right)$. In this case, it is easy to show that for all $x \in \mathcal{X} \backslash(2 B)$ and $y \in B, d(x, y)<\epsilon$. Thus, by the vanishing moment of $b$ with (3.2) and (1.4), we easily see that

$$
\begin{aligned}
\mathrm{F}_{3} & \lesssim \int_{\mathcal{X} \backslash(2 B)} \int_{\mathcal{X}}\left|K(x, y)-K\left(x, x_{B}\right)\right||b(y)| d \mu(y) d \mu(x) \\
& \lesssim \int_{\mathcal{X} \backslash(2 B)} \int_{\mathcal{X}}\left[\frac{d\left(y, x_{B}\right)}{d\left(x, x_{B}\right)}\right]^{\tau} \frac{|b(y)|}{\lambda(x, d(x, y))} d \mu(y) d \mu(x) \\
& \lesssim \sum_{j=1}^{2}\left|\kappa_{j}\right| \int_{\mathcal{X} \backslash(2 B)} \int_{B_{j}}\left[\frac{r_{B}}{d\left(x, x_{B}\right)}\right]^{\tau} \frac{\left|a_{j}(y)\right|}{\lambda\left(x_{B}, d\left(x, x_{B}\right)\right)} d \mu(y) d \mu(x) \\
& \lesssim \sum_{j=1}^{2}\left|\kappa_{j}\right| \sum_{k=1}^{\infty} \int_{\left(2^{k+1} B\right) \backslash\left(2^{k} B\right)}\left[\frac{r_{B}}{2^{k} r_{B}}\right]^{\tau} \frac{d \mu(x)}{\lambda\left(x_{B}, d\left(x, x_{B}\right)\right)} \lesssim \sum_{j=1}^{2}\left|\kappa_{j}\right| .
\end{aligned}
$$

Case (ii). $\epsilon \in\left[r_{B}, \infty\right)$. In this case, we first write

$$
\begin{aligned}
\mathrm{F}_{3} \leq & \int_{d\left(x, x_{B}\right)>r_{B}+\epsilon} \int_{d(x, y)>\epsilon}\left|K(x, y)-K\left(x, x_{B}\right)\right||b(y)| d \mu(y) d \mu(x) \\
& +\int_{2 r_{B} \leq d\left(x, x_{B}\right) \leq r_{B}+\epsilon} \int_{d(x, y)>\epsilon}|K(x, y)||b(y)| d \mu(y) d \mu(x)=: \mathrm{F}_{3,1}+\mathrm{F}_{3,2} .
\end{aligned}
$$

For the term $\mathrm{F}_{3,1}$, notice that for all $x \in \mathcal{X}$ such that $d\left(x, x_{B}\right)>r_{B}+\epsilon$ and all $y \in B$, $d(x, y)>\epsilon$. Thus, by the same argument as to the Case (i), we conclude that

$$
\mathrm{F}_{3,1} \lesssim \sum_{j=1}^{2}\left|\kappa_{j}\right| .
$$

On the other hand, from (3.1), (1.3) and (1.4), it follows that

$$
\begin{aligned}
\mathrm{F}_{3,2} & \lesssim \sum_{j=1}^{2}\left|\kappa_{j}\right| \int_{d\left(x, x_{B}\right) \leq 2 \epsilon} \int_{d(x, y)>\epsilon} \frac{1}{\lambda(x, d(x, y))}\left|a_{j}(y)\right| d \mu(y) d \mu(x) \\
& \lesssim \sum_{j=1}^{2}\left|\kappa_{j}\right| \int_{d\left(x, x_{B}\right) \leq 2 \epsilon} \frac{1}{\lambda\left(x_{B}, \epsilon\right)} d \mu(x)\left\|a_{j}\right\|_{L^{1}(\mu)} \lesssim \sum_{j=1}^{2}\left|\kappa_{j}\right| .
\end{aligned}
$$


Combining the estimates for $\mathrm{F}_{3,1}$ and $\mathrm{F}_{3,2}$, we see that

$$
\mathrm{F}_{3} \lesssim \sum_{j=1}^{2}\left|\kappa_{j}\right|
$$

in this case.

The estimates for $F_{1}, F_{2}$ and $F_{3}$ imply (3.12). Thus, $(v) \Rightarrow$ (i), which further implies that (i) - (vi) are equivalent.

(vi) $\Leftrightarrow$ (vii). The implication (vii) $\Rightarrow$ (vi) is obvious. Let us prove (vi) $\Rightarrow$ (vii). Indeed, based on the equivalence of (i)-(vi) proved above, we conclude that if (vi) holds, then $T$ is bounded from $H^{1}(\mu)$ into $L^{1}(\mu)$ and from $L^{\infty}(\mu)$ into $\operatorname{RBMO}(\mu)$. By the interpolation result [1, Theorem 6.4], we see that $T$ is bounded for all $p \in(1, \infty)$. This means that (vii) holds. Thus, we proved that (vi) $\Leftrightarrow$ (vii), which completes the proof of Theorem 3.1.

Proof of Corollary 3.1. By Theorem 3.1, the proof of Corollary 3.1 is reduced to proving that Theorem 3.1(iv) is true. Let $B$ be a fixed ball and $f$ a bounded function with support contained in $B$. By the homogeneity of $\|\cdot\|_{\operatorname{RBMO}(\mu)}$, we may assume that $\|f\|_{L^{\infty}(\mu)}=1$. By some trivial computation, we easily see that for any $\sigma \in(0,1)$,

$$
\begin{aligned}
\int_{B}\left|T_{\epsilon}(f)(x)\right|^{\sigma} d \mu(x) & =\sigma \int_{0}^{1} t^{\sigma-1} \mu\left(\left\{x \in B:\left|T_{\epsilon}(f)(x)\right|>t\right\}\right) d t+\sigma \int_{1}^{\infty} \cdots \\
& \lesssim \mu(B)+\int_{1}^{\infty} t^{\sigma-1} \Phi\left(\frac{1}{t}\right) d t \int_{B} \Phi(|f(x)|) d \mu(x) \lesssim \mu(B),
\end{aligned}
$$

which completes the proof of Corollary 3.1.

Acknowledgements. The authors would like to express their deep thanks to the referee for his/her very careful reading and many valuable suggestive remarks which essentially improve the presentation of the article.

\section{References}

[1] AnH, B. T., and X. T. Duong: Hardy spaces, regularized BMO spaces and the boundedness of Calderón-Zygmund operators on non-homogeneous spaces. - J. Geom. Anal., 2011, DOI: 10.1007/s12220-011-9268-y.

[2] Coifman, R. R., and G. Weiss: Analyse harmonique non-commutative sur certains espaces homogénes. - Lecture Notes in Math. 242, Springer, Berlin, 1971.

[3] Heinenon, J.: Lectures on analysis on metric spaces. - Springer-Verlag, New York, 2001.

[4] HU, G., and D. YANG: Weighted norm inequalities for maximal singular integrals with nondoubling measures. - Studia Math. 187, 2008, 101-123.

[5] Hu, G., X. WANG, and D. YANG: A new characterization for regular BMO with non-doubling measures. - Proc. Edinb. Math. Soc. (2) 51, 2008, 155-170.

[6] Hu, G., D. YAng, and D. YAnG: A new characterization of $\operatorname{RBMO}(\mu)$ by John-Strömberg sharp maximal functions. - Czechoslovak Math. J. 59, 2009, 159-171.

[7] Hytönen, T.: A framework for non-homogeneous analysis on metric spaces, and the RBMO space of Tolsa. - Publ. Mat. 54, 2010, 485-504.

[8] Hytönen, T., and H. Martikainen: Non-homogeneous $T b$ theorem and random dyadic cubes on metric measure spaces. - J. Geom. Anal. 22, 2012, 1071-1107. 
[9] Hytönen, T., D. YAng, and D. YAng: The Hardy space $H^{1}$ on non-homogeneous metric spaces. - Math. Proc. Cambridge Philos. Soc. 153, 2012, 9-31.

[10] John, F.: Quasi-isometric mappings. - In: Seminari 1962/63 Anal. Alg. Geom. e Topol., vol. 2, Ist. Naz. Alta Mat., Ediz. Cremonese, Rome, 1965, 462-473.

[11] Journé, J.-L.: Calderón-Zygmund operators, pseudodifferential operators and the Cauchy integral of Calderón. - Lecture Notes in Math. 994, Springer-Verlag, Berlin, 1983.

[12] Lerner, A. K.: On the John-Strömberg characterization of BMO for nondoubling measures. - Real Anal. Exchange 28, 2002, 649-660.

[13] Lin, H., and D. YANG: Spaces of type BLO on non-homogeneous metric measure spaces. Front. Math. China 6, 2011, 271-292.

[14] Lin, H., and D. YANG: An interpolation theorem for sublinear operators on non-homogeneous metric measure spaces. - Banach J. Math. Anal. 6, 2012, 168-179.

[15] LiU, S., D. YANG, and D. YAng: Boundedness of Calderón-Zygmund operators on nonhomogeneous metric measure spaces: equivalent characterizations. - J. Math. Anal. Appl. 386, $2012,258-272$.

[16] LuUkkainen, J., and E. Saksman: Every complete doubling metric space carries a doubling measure. - Proc. Amer. Math. Soc. 126, 1998, 531-534.

[17] Nazarov, F., S. Treil, and A. Volberg: Weak type estimates and Cotlar inequalities for Calderón-Zygmund operators on nonhomogeneous spaces. - Int. Math. Res. Not. IMRN 9, 1998, 463-487.

[18] Nazarov, F., S. Treil, and A. Volberg: The $T b$-theorem on non-homogeneous spaces. Acta Math. 190, 2003, 151-239.

[19] Strömberg, J. O.: Bounded mean oscillation with Orlicz norms and duality of Hardy spaces. - Indiana Univ. Math. J. 28, 1979, 511-544.

[20] Tolsa, X.: BMO, $H^{1}$ and Calderón-Zygmund operators for non doubling measures. - Math. Ann. 319, 2001, 89-149.

[21] Tolsa, X.: Littlewood-Paley theory and the $T(1)$ theorem with non-doubling measures. Adv. Math. 164, 2001, 57-116.

[22] Tolsa, X.: The space $H^{1}$ for nondoubling measures in terms of a grand maximal operator. Trans. Amer. Math. Soc. 355, 2003, 315-348.

[23] Tolsa, X.: Painlevé's problem and the semiadditivity of analytic capacity. - Acta Math. 190, 2003, 105-149.

[24] TolsA, X.: Analytic capacity and Calderón-Zygmund theory with non doubling measures. In: Seminar of Mathematical Analysis, Colecc. Abierta 71, Univ. Sevilla Secr. Publ., Seville, 2004, 239-271.

[25] VolBerg, A., and B. D. Wick: Bergman-type singular operators and the characterization of Carleson measures for Besov-Sobolev spaces on the complex ball. - Amer. J. Math. (to appear), arXiv: 0910.1142 . 